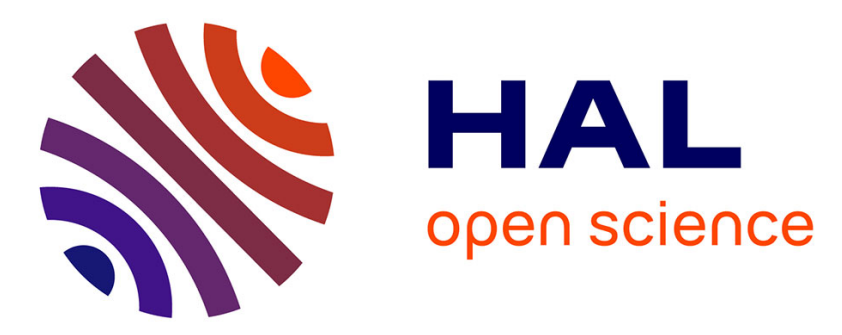

\title{
Linearized global and local buckling analysis of sandwich struts with a refined quasi-3D model
}

\author{
M. d'Ottavio, O. Polit
}

\section{To cite this version:}

M. d'Ottavio, O. Polit. Linearized global and local buckling analysis of sandwich struts with a refined quasi-3D model. Acta Mechanica, 2015, 226 (1), pp.81-101. 10.1007/s00707-014-1169-2 . hal01367047

\section{HAL Id: hal-01367047 \\ https://hal.science/hal-01367047}

Submitted on 9 Jan 2019

HAL is a multi-disciplinary open access archive for the deposit and dissemination of scientific research documents, whether they are published or not. The documents may come from teaching and research institutions in France or abroad, or from public or private research centers.
L'archive ouverte pluridisciplinaire HAL, est destinée au dépôt et à la diffusion de documents scientifiques de niveau recherche, publiés ou non, émanant des établissements d'enseignement et de recherche français ou étrangers, des laboratoires publics ou privés. 


Michele D'Ottavio · Olivier Polit

\section{Linearized global and local buckling analysis of sandwich struts with a refined quasi-3D model}

\section{Introduction}

Sandwich panels are fundamental structural elements of many industrial applications with sharp weight constraints, in particular for aerospace and naval components [1]. They basically consist of two thin and stiff load carrying face sheets (the skins) that are separated by a thick and relatively weak core made of lowdensity material. The core enhances the bending rigidity of the panel, transfers the load between the face sheets essen-tially by transverse shear and normal stresses and may additionally act as thermal insulator, vibration damper or sound absorber [2]. Due to the very different mechanical properties and geometrical dimensions of skins and core, the failure behavior of sandwich panels can be very different from that of other composite structures $[3,4]$. Common failure modes of sandwich panels can be classified into face sheet fracture (e.g., impact in compos-ite skins), core shear failure or failure modes due to compressive stress. The latter are principally related to instability phenomena and can involve the whole sandwich panel (overall buckling), mainly the core (shear crimping) or mainly the face sheets (local buckling). In laminated composite face sheets, microbuckling may additionally appear [5-7]. Overall buckling and shear crimping are usually referred to as global instabilities, even if the wavelength of the crimping mode is very small because the transverse shear modulus of the core is very low $[8,9]$. On the other hand, the length scale of the local buckling pattern is of the order of the panel thickness or even smaller: In honeycomb cores, the compressed face sheet may locally buckle inside the core's cells (dimpling), whereas in homogeneous core materials (e.g., foams or balsa wood), the compressed face sheet typically forms "wrinkles" whose wavelength may be comparable to a fraction of the core thickness [10]. Besides the characteristic stiffness loss of buckled configurations, wrinkling can promote localized skin-core

M. D'Ottavio ( $\varangle) \cdot$ O. Polit

Université Paris Ouest, LEME EA4416, 50 rue de Sèvres, 92410 Ville d'Avray, France

E-mail: michele.d_ottavio@u-paris10.fr

Tel.: +33-1-40-97-48-84 
debonding (delamination buckling) or local core crushing (crippling) [11,12]. In practice, these various failure modes do often interact. For instance, when the overall buckling and wrinkling loads are of the same order of magnitude, the compressed face sheet in the overall buckled configuration may additionally wrinkle inside the core, which may lead to a localized failure [13-16]. The analysis of both global and local buckling behavior of sandwich panels appears thus as a necessary design step. The difference between the length scales of these failure modes represents a major issue for their computational prediction $[17,18]$.

Compression instability problems are classically treated by linearized bifurcation buckling analysis, which reduces the computation of the critical load to the solution of an eigenvalue problem, see e.g., [19]. This simplified approach is only valid if the panel remains flat under the action of the initial in-plane loading. Therefore, it disregards the effects of small perturbations (imperfections) of the nominal initial configuration, which can drastically reduce the critical loads. The imperfection sensitivity of compressed sandwich structures has been early recognized in [20] and taken into account in a number of works by referring to the nonlinear equilibrium and stability equations, e.g., in [21-25]. Nevertheless, in order to circumvent complex and expensive solutions of the nonlinear equations, practical design methods cut down the bifurcation loads by pertinent knockdown factors, see e.g. [9]. The present work follows this approach and focuses on the linearized bifurcation buckling analysis of perfectly straight sandwich panels under uniaxial compression (struts).

This paper is organized as follows: Sect. 1.1 proposes a detailed review of the models employed for capturing overall buckling and wrinkling before discussing the scope of the present work in Sect. 1.2. The theoretical development of the present model is presented in Sect. 2 with due details concerning the proposed application to the linearized bifurcation analysis of flat and symmetric sandwich structures. Numerical solutions for various case studies are discussed in Sect. 3, and the main conclusions are finally summarized in Sect. 4.

\subsection{Models for overall buckling and wrinkling of sandwich struts}

For the literature review of this section, the most popular notation is adopted as illustrated in Fig. 1. The sandwich strut is a flat rectangular panel of length $a$ along $x$, thickness $h$ along $z$, width $b$ along $y$, that is compressed along the $x$ direction. Symmetric sandwich sections will be considered, which consist of a core of thickness $h_{\mathrm{c}}$ as well as top and bottom face sheets of equal thickness $h_{\mathrm{f}}$ and made of the same material (throughout the paper, subscript or superscript $f$ and $c$ stay for face sheet and core, respectively).

Due to the very different length scales of the buckled configuration, two separated approaches are classically employed to predict overall and local buckling, see, for instance, the comprehensive monograph of Allen [26] or the more recent book by Carlsson and Kardomateas [3]. In the following, several models are reviewed according to this classification, where special attention is paid to the underlying hypotheses.

Overall buckling The classical Euler formula for a narrow strut (plane stress in $x z$ plane) whose cross-section is symmetric and composed as in Fig. 1 by two materials with axial Young's moduli $E_{x}^{\mathrm{f}}$ and $E_{x}^{\mathrm{c}}$ reads

$$
P_{\mathrm{E}}=\frac{\pi^{2} b}{a^{2}}\left[E_{x}^{\mathrm{f}} h_{\mathrm{f}}\left(\frac{h_{\mathrm{f}}^{2}}{6}+\frac{\left(h_{\mathrm{f}}+h_{\mathrm{c}}\right)^{2}}{2}\right)+E_{x}^{\mathrm{c}} \frac{h_{\mathrm{c}}^{3}}{12}\right] .
$$

This equation fails to provide a conservative estimate of the bifurcation buckling load because it neglects the relevant transverse shear deformation of the core. Classical overall buckling formulas for sandwich struts are associated with Engesser's formula, which reduces the classical Euler load by retaining the transverse shear deformation in the soft core [27,28]. Under the hypotheses of a soft core $\left(E_{x}^{\mathrm{c}}<<E_{x}^{\mathrm{f}}\right)$ and thin face beams $\left(h_{\mathrm{f}} / h_{\mathrm{c}}<0.2\right)$, Allen suggests the following expression for the overall buckling stress [26]:

$$
\sigma_{\mathrm{cr}}^{A 1}=\frac{P_{A 1}}{2 b h_{\mathrm{f}}}=\frac{1}{2 b h_{\mathrm{f}}}\left[\frac{1}{P_{\mathrm{E} 1}}+\frac{1}{P_{\mathrm{c}}}\right]^{-1},
$$

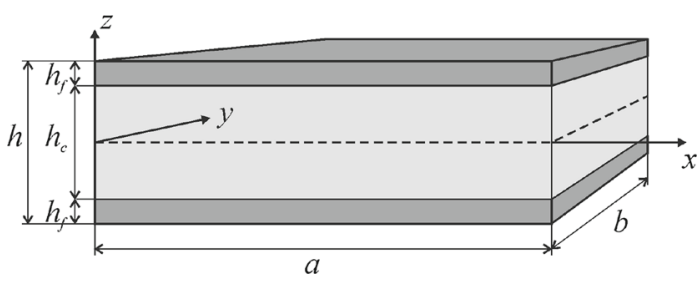

Fig. 1 Geometric parameters and axis notation for the sandwich plate with symmetric cross-section 
Antisymmetric mode

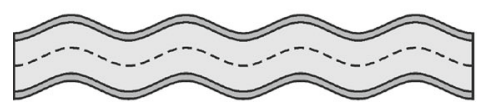

Symmetric mode

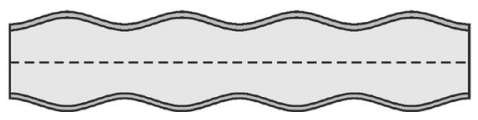

Single-sided wrinkling

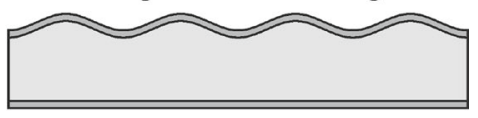

Fig. 2 The three wrinkling modes of a sandwich panel

where $P_{\mathrm{E} 1}$ is an approximation of Eq. (1) which includes the soft core and thin face beam hypotheses:

$$
P_{\mathrm{E} 1}=\frac{\pi^{2} b}{2 a^{2}} E_{x}^{\mathrm{f}} h_{\mathrm{f}}\left(h_{\mathrm{c}}+h_{\mathrm{f}}\right)^{2},
$$

and $P_{\mathrm{c}}$ is the shear crimping load that depends on the transverse shear modulus $G_{x z}$ and is defined by

$$
P_{\mathrm{c}}=G_{x z}^{\mathrm{c}} b \frac{\left(h_{\mathrm{c}}+h_{\mathrm{f}}\right)^{2}}{h_{\mathrm{c}}} \approx G_{x z}^{\mathrm{c}} b h_{\mathrm{c}} .
$$

Note that Euler's loads $P_{\mathrm{E}}$ and $P_{\mathrm{E} 1}$ have been written for a simply supported beam and are the only terms that depend on the boundary conditions. If the face beams are thick, their second area moment should be retained, which leads to the following overall buckling stress formulated by Allen [26]:

$$
\sigma_{\mathrm{cr}}^{\mathrm{A} 2}=\frac{P_{\mathrm{A} 2}}{2 b h_{\mathrm{f}}}=\frac{P_{\mathrm{E} 2}}{2 b h_{\mathrm{f}}}\left[\frac{1+\frac{P_{\mathrm{Ef}}}{P_{\mathrm{c}}}-\frac{P_{\mathrm{Ef}}}{P_{\mathrm{c}}} \frac{P_{\mathrm{Ef}}}{P_{\mathrm{E} 2}}}{1+\frac{P_{\mathrm{E} 2}}{P_{\mathrm{c}}}-\frac{P_{\mathrm{Ef}}}{P_{\mathrm{c}}}}\right]
$$

where $P_{\mathrm{c}}$ is the shear crimping load given in Eq. (2.3), and

$$
P_{\mathrm{E} 2}=\frac{\pi^{2} b}{a^{2}}\left[E_{x}^{\mathrm{f}} h_{\mathrm{f}}\left(\frac{h_{\mathrm{f}}^{2}}{6}+\frac{\left(h_{\mathrm{f}}+h_{\mathrm{c}}\right)^{2}}{2}\right)\right] \text { and } P_{\mathrm{Ef}}=\frac{\pi^{2} b}{a^{2}} E_{x}^{\mathrm{f}} \frac{h_{\mathrm{f}}^{3}}{6} .
$$

Note that $P_{\mathrm{Ef}}$ corresponds to Euler's load of the simply supported face beams behaving as independent struts (i.e., without the presence of the core). If the sandwich strut is wide, the axial Young's moduli in the above formulas should account for the plane strain assumption in order to reproduce the cylindrical bending state. Local buckling (wrinkling) Due to the transverse deformability of the soft core, the thin face sheets may locally buckle into the core, thus forming short-wavelength wrinkles while the whole sandwich strut remains stable. The standard classification of antisymmetric and symmetric wrinkling modes, illustrated in Fig. 2, is based on the local deformation pattern of the wrinkled configuration [26]. In antisymmetric wrinkling, the top and bottom face sheets buckle in-phase, which produces a wrinkled mid-surface of the core; in symmetric wrinkling, the top and bottom face sheets buckle out-of-phase, and the core undergoes essentially a transverse normal deformation while its mid-surface remains straight; the single-sided wrinkling mode concerns the local instability of only one face sheet, no interaction occurs with the other face sheet which may remain flat. The two former modes can appear in sandwich panels whose face sheets are equally compressed, single-sided wrinkling may occur in the compressed face of a bent panel or in the thinner face sheet of an unsymmetric sandwich strut.

The classical analysis of wrinkling addresses the instability of a strut (the face sheet) that is elastically supported by the core. By supposing the wrinkling wavelength to be very short, the actual dimension of the panel as well as its end conditions is disregarded: Wrinkling is thus treated as a material instability of the sandwich structure rather than a structural instability. The face sheets are usually modeled as thin beams by referring to Euler-Bernoulli's theory in which transverse shear is neglected, see e.g., [29,30]. Due to their much higher stiffness compared with the core, the compressive load is assumed to be carried only by the face sheets. Since the face sheets are supposed to deform only in bending, that is, no axial rigidity is accounted for, this modeling approach is not applicable to the global buckling.

A large variety of models for the elastic support provided by the core has been proposed. The simplest model is that associated with Winkler's elastic foundation, according to which the core is modeled as closely spaced compression springs whose stiffness depends on the core's transverse normal Young's modulus $E_{z}^{\mathrm{c}}$ and its thickness $h_{\mathrm{c}}$. For symmetric wrinkling, the critical stress according to Winkler's model reads

$$
\sigma_{\mathrm{cr} W}=\sqrt{\frac{2 h_{\mathrm{f}}}{3 h_{\mathrm{c}}}} \sqrt{E_{x}^{\mathrm{f}} E_{z}^{\mathrm{c}}} .
$$


Winkler's model is not suitable to predict antisymmetric wrinkling because the in-phase waves of the face sheets do not stretch nor compress the core but essentially shear it. The absence of transverse shear limits the range of applicability of this simple model to rather long wavelengths and, equivalently, thin cores. Enhanced elastic foundation models have been employed by referring to two-parameter models which include the transverse shear deformation and are hence applicable to local bending and short-wavelength wrinkling problems [31-34].

Other models have been proposed in which the core is considered as a continuous isotropic or orthotropic medium. Among them, one may identify anti-plane core models, in which the core has only transverse stiffness $E_{z}^{\mathrm{c}}$ and $G_{x z}^{\mathrm{c}}$ and the in-plane stiffness is neglected $\left(E_{x}^{\mathrm{c}}=0\right)[35,36]$, and models including a finite value for the in-plane stiffness $[20,26,29,30]$. Furthermore, several models are limited to isotropic cores $[26,29,36]$, while others include material orthotropy $[20,30,35]$. Finally, one may identify core models that account for the interaction between the top and bottom face sheets (finite thickness) $[20,29,35]$ from those representing a core of infinite thickness, in which the interaction between the face sheets is disregarded $[30,36]$. A widely accepted expression for the wrinkling stress obtained from these models can be written as

$$
\sigma_{\mathrm{cr}}=C \sqrt[3]{E_{x}^{\mathrm{f}} E_{z}^{\mathrm{c}} G_{x z}^{\mathrm{c}}}
$$

In practical design, knockdown factors are usually employed which let $C$ take a value of 0.5 [8] or 0.44 [37]. In general, $C$ depends on the face-to-core thickness ratio as well as on the material properties of both materials. As the thickness of the core increases, the function $C$ admits an asymptotic value that depends on the model but is independent of the wrinkling mode, see e.g., $[20,26,29]$; in fact, if the core is "sufficiently thick", there is no interaction between the face sheets, and hence, the wrinkling mode separation disappears [23]. Note that the interaction between top and bottom faces is not only a matter of "geometric distance" but it is actually related to the wavelength of the wrinkling response, which in turns depends on geometric and material properties: The interaction is more pronounced for long wavelengths, whereas short wavelengths damp out more rapidly inside the core. These relations appear explicitly in the formulations of "decay" models, see e.g., $[26,35,36]$.

For completeness, the problem of wrinkling due to biaxial loading is briefly addressed. It requires some more involved analyses because the critical stress depends not only on the wavelength but also on the direction of the wrinkles, which in general are not aligned with principal material or geometrical directions $[38,39]$.

General models for overall and local buckling Several "unified" models have been proposed that are capable of representing both the overall buckling and wrinkling instabilities [40]. In these models, the core is an elastic medium of finite thickness which links the face sheets by some continuity conditions that depend on the modeling technique and take into account the symmetry or antisymmetry of the buckling mode. Most of these models employ Euler-Bernoulli's thin beam theory for the face sheets. For the model to be capable of representing both global buckling and wrinkling, it is essential that the face sheets have both axial and bending stiffness. The initial load is supposed to be carried entirely by the stiff face sheets, and von Kàrmàn's assumption is often adopted for the nonlinear formulation of the beam kinematics. Different approaches have been used for representing the core's behavior, all of which retain the transverse normal deformation in order to capture the wrinkling modes. A common assumption makes use of anti-plane cores, which disregards the in-plane load carrying capability of the core [21,41-43]. This hypothesis has been shown to give misleading results not only when the core is isotropic [44], but also when the core is highly orthotropic, e.g., for honeycomb cores [30]. In [45], the core has been modeled as a 2D isotropic continuum (plane stress setting for narrow beams), instead of resorting to a kinematic model as done in the previously cited works. A refined plate model originally formulated for composite laminates has been shown to grasp both overall and local buckling modes of sandwich struts [46]. Higher-order polynomials are used for describing the kinematics in each constituting layer of both the skins and the core, and no simplifying assumptions concerning the core's material or the mode shape are introduced. While an excellent accuracy with respect to available elasticity solutions could be demonstrated for the overall buckling loads, no 3D wrinkling results have been taken for comparison.

Elasticity solutions Reference elasticity solutions to some linear problems of sandwich panels, including buckling, have been provided by Meyer-Piening [47,48]. More recently, several papers focused on overall buckling and wrinkling of wide sandwich struts within an elasticity approach in the 2D plane strain setting [44, 49-51]. While the models in [44,51] are formulated for isotropic materials only, those in [49,50] are formulated for orthotropic face and core materials. In these works, initial loads are introduced by an applied uniform axial strain acting across the whole sandwich cross-section, which contrasts with most of the literature on wrinkling analysis. The analysis in [50] has shown that, if the core does not carry the initial compressive load, a nonperiodic edge buckling instability is likely to occur at lower loads than the periodic wrinkling. Von Kàrmàn's small strains/large rotations assumption, as originally employed for the thin skins, has been used in the elasticity 
approach proposed in [44,49] as well. However, the paper [51] demonstrated that a nonlinear strain definition that neglects the axial shortening due to the initial stress can overestimate the buckling loads of short-wavelength modes, for which the "thinness" assumption is no longer valid. This error is shown to increase if, additionally, an inconsistent conjugated stress definition is employed, as it is actually done in $[44,49]$ and even in commercial finite element (FE) codes [51]. These issues are avoided by a consistent nonlinear formulation that employs constant elastic moduli, the Green-Lagrange strains and Trefftz incremental stresses [51]. Such a formulation has been recently used for a 2D plane strain elasticity approach to a linearized stability analysis of sandwich structures that was, however, limited to the overall buckling [28].

\subsection{Scope of the present work}

The present paper employs an established higher-order composite plate model to analyze both overall buckling and wrinkling of sandwich panels under uniaxial compression. The model is based on a partially mixed variational equation proposed by Reissner that permits to introduce independent assumptions for the displacement and the transverse stress fields $[52,53]$. This approach can, hence, easily and exactly satisfy the interface continuity conditions of a perfect bond. The six unknown functions (three displacements $u_{i}$ and three transverse stresses $\sigma_{i 3}$ ) are then interpolated by a fourth-order polynomial inside each layer. The layer-wise description permits to further refine the model by simply increasing the number of numerical layers the composite crosssection is divided into. This model, first proposed by Carrera, is commonly referred to as LM4 (Layer-wise, Mixed, 4th order polynomial) and has proved to yield quasi-3D solutions for various problems of composite and sandwich panels involving global and local response [54-58]. The model has been successfully extended and applied to the linearized buckling analysis of laminated composite shells [59]. Both von Kàrmàn and Green-Lagrange finite strain definitions have been included, and the pre-buckling loading has been defined in terms of either an initial stress or an initial strain field. The initial load can be further applied to the whole sandwich cross-section or to the face sheets only. Navier's closed-form solution is used to solve the resulting $2 \mathrm{D}$ governing equations in a strong form.

Due to the intrinsic limitations of the adopted 2D modeling and solution approach, this paper limits the scope to periodic buckling modes with buckle wavelengths that are parallel to the plate and orthotropy axes and constant for the whole cross-section. Therefore, several relevant aspects of practical applications are disregarded, such as non-periodic patterns, wrinkling under biaxial load, single-sided wrinkling, global/local buckling interaction or the role of initial imperfections. Finally, since the aim is to provide some reference solutions that may be useful for validating approximated models, the results of the linearized buckling analysis will not be modified by knockdown factors. The influence of several parameters will be addressed: plate slenderness, face-to-core thickness ratio, face and core stiffness, material orthotropy, von Kàrmàn nonlinearities and initial load definition.

\section{Problem definition and modeling}

We consider a symmetric sandwich plate composed of $N_{l}$ homogeneous, orthotropic and perfectly bonded layers. The central layer is the core of thickness $h_{\mathrm{c}}$, and the outer face sheets of thickness $h_{\mathrm{f}}$ may be composite laminates. In the following, Cartesian coordinates will be denoted $\left(x_{\alpha}, x_{3}=z\right)$, where $\alpha=1,2$ refers to the in-plane directions. This index notation is more convenient for the formulation of the computational plate model, in which the transverse direction $x_{3}=z$ is treated differently from the in-plane directions $x_{\alpha}$. The plate is hence defined as the volume $V=\Omega \times\left[-\frac{h}{2}, \frac{h}{2}\right]$, where $\Omega=[0, a] \times[0, b]$ is the reference surface in the $x_{\alpha}$ plane and $h=h_{\mathrm{c}}+2 h_{\mathrm{f}}$ the constant thickness along the transverse direction $x_{3}=z$, see also Fig. 1 . Each layer $k=1,2, \ldots N_{l}$ of the sandwich structure is identified by the location along the coordinate $z$ of its reference surface $\Omega_{k}$ and its thickness $h_{k}$.

\subsection{Governing equations for the linearized stability analysis}

The buckling equations are obtained in the framework of the linearized stability analysis of a body subjected to initial stresses [60]. A variational formulation is used in order to derive a reduced 2D model by introducing assumptions for the through-thickness distribution of the field variables. The critical load is then given by the scalar parameter $\lambda$ that multiplies the initial stress state $\sigma_{i j}^{0}$ and that satisfies the variational equation 


$$
\int_{V} \delta \epsilon_{i j} \sigma_{i j}+\left(\delta u_{m},{ }_{j}\right) \lambda \sigma_{i j}^{0} u_{m}, i \mathrm{~d} V=0,
$$

where $\delta$ denotes virtual variation, and Einstein's summation convention is employed with $i, j, m=1,2,3$. By introducing the Green-Lagrange strains

$$
E_{i j}=\epsilon_{i j}+e_{i j}
$$

as the sum of the linear contribution

$$
\epsilon_{i j}=\frac{1}{2}\left(u_{i},{ }_{j}+u_{j, i}\right)
$$

and of the nonlinear terms

$$
e_{i j}=\frac{1}{2} u_{m},{ }_{i} u_{m},{ }_{j}
$$

the variational equation can be reformulated as

$$
\int_{V} \delta \epsilon_{i j} \sigma_{i j}+\delta e_{i j} \lambda \sigma_{i j}^{0} \mathrm{~d} V=0 .
$$

The displacement-based functional Eq. (8) is next augmented as suggested by Reissner [52] in order to construct a $2 \mathrm{D}$ model that accounts in a natural manner for the transverse stress continuity conditions at the interfaces of a heterogeneous plate. Reissner's partially mixed formulation is hence extended to include the initial stress field for the buckling analysis:

$$
\int_{V} \delta \epsilon_{\alpha \beta}^{G} \sigma_{\alpha \beta}^{C}+\delta \epsilon_{i 3}^{G} \sigma_{i 3}^{M}+\delta \sigma_{i 3}^{M}\left(\epsilon_{i 3}^{G}-\epsilon_{i 3}^{C}\right)+\delta e_{i j}^{G} \lambda \sigma_{i j}^{0} \mathrm{~d} x_{i}=0,
$$

with $\alpha, \beta=1,2$. The superscript $G$ indicates that the strain is defined from the geometric relations Eq. (7), superscript $M$ that the transverse stress field is independently assumed (modeled) - see Sect. 2.2, and superscript $C$ that the field variable is obtained from the mixed form of the constitutive equation

$$
\left[\begin{array}{c}
\sigma_{\alpha \beta}^{C} \\
\epsilon_{i 3}^{C}
\end{array}\right]=\left[\begin{array}{ll}
\tilde{C}_{\alpha \beta \alpha \beta} & \tilde{C}_{\alpha \beta i 3} \\
\tilde{C}_{i 3 \alpha \beta} & \tilde{C}_{i 3 i 3}
\end{array}\right]\left[\begin{array}{c}
\epsilon_{\alpha \beta}^{G} \\
\sigma_{i 3}^{M}
\end{array}\right] .
$$

The Euler equations associated with Eq. (9) are the three equilibrium equations of the perturbed configuration (Trefftz formulation)

$$
\delta u_{i}: \frac{\partial}{\partial x_{j}}\left[\sigma_{i j}+\sigma_{m j}^{0} u_{i}, m\right]=0,
$$

and the three compatibility conditions for the transverse strains

$$
\delta \sigma_{i 3}: \quad \epsilon_{i 3}^{G}-\epsilon_{i 3}^{C}=\epsilon_{i 3}^{G}-\left(\tilde{C}_{i 3 \alpha \beta} \epsilon_{\alpha \beta}^{G}+\tilde{C}_{i 3 i 3} \sigma_{i 3}^{M}\right)=0 .
$$

It is emphasized that this formulation, based on Green-Lagrange nonlinear strains and elastic coefficients that are constant throughout the perturbation process, is a consistent incremental formulation for sandwich structures with shear-compliant cores [27,51].

In view of the subsequent introduction of the $2 \mathrm{D}$ approximation for the multilayered plate, see Sect. 2.2, the volume integral in Eq. (9) is split in a surface integral over $\Omega$ and the integral over the cross-section $h$, that is in turn decomposed in $N_{l}$ integrals over each layer's thickness $h_{k}$, viz.

$$
\int_{V}(\ldots) \mathrm{d} x_{i}=\int_{\Omega}\left\{\bigcup_{k=1}^{N_{l}} \int_{h_{k}}(\ldots) \mathrm{d} z\right\} \mathrm{d} x_{\alpha} .
$$




\subsubsection{Initial stress fields}

The above general formulation is next specialized to the examined cases for which the initial stress in each layer $k$ is limited to direct stress components, i.e., $\sigma_{i j}^{0 k}=0$ for $i \neq j$. Furthermore, only a membrane preload will be considered, i.e., $\sigma_{33}^{0 k}=0$. Note that this condition respects the stress-free condition on the outer faces of the sandwich plate. Under these circumstances, Eq. (9) reduces to

$$
\int_{\Omega}\left\{\bigcup_{k=1}^{N_{l}} \int_{h_{k}} \delta \epsilon_{\alpha \beta}^{G k} \sigma_{\alpha \beta}^{C k}+\delta \epsilon_{i 3}^{G k} \sigma_{i 3}^{M k}+\delta \sigma_{i 3}^{M k}\left(\epsilon_{i 3}^{G k}-\epsilon_{i 3}^{C k}\right)+\delta e_{\alpha \alpha}^{G k} \lambda \sigma_{\alpha \alpha}^{0 k} \mathrm{~d} z\right\} \mathrm{d} x_{\alpha}=0,
$$

with the virtual nonlinear strains defined according to Eq. (7) as

$$
\delta e_{\alpha \alpha}^{G k}=\left(u_{1, \alpha}^{k} \delta u_{1}^{k}, \alpha\right)+\left(u_{2}^{k}, \alpha \delta u_{2}^{k}, \alpha\right)+\left(u_{3, \alpha}^{k} \delta u_{3, \alpha}^{k}\right) .
$$

In this work, only uniaxial compression shall be considered, and the initial stress field $\sigma_{11}^{0 k}$ in the $k$ th layer will be defined in two alternate manners:

- Uniform stress in the face sheets: $\sigma_{11}^{0 k}=\sigma_{11}^{0}$ if the $k$ th layer belongs to a face sheet, while $\sigma_{11}^{0 k}=0$ if the $k$ th layer belongs to the core (i.e., the core carries no initial load).

- Uniform strain: The whole cross-section is uniformly strained by $\varepsilon_{11}^{0}$. The initial stress state is hence uniform in each layer and proportional to the elastic stiffness of the layer: $\sigma_{11}^{0 k}=C_{11}^{k} \varepsilon_{11}^{0}$, where $C_{11}^{k}$ is the stiffness coefficient of the $k$ th layer.

\subsubsection{Von Kàrmàn's approximation}

Instead of employing the finite Green-Lagrange strains defined in Eq. (7), geometrically nonlinear plate models often refer to von Kàrmàn's approximation, which takes into account large deflections but still within a small material strain range [61]. Hence, von Kàrmàn's nonlinear strains retain only the nonlinear terms associated with the transverse displacement $u_{3}$, which reduces the in-plane contributions of Eq. (7.3) to

$$
e_{\alpha \alpha(v K)}=\frac{\left(u_{3}, \alpha\right)^{2}}{2} \text {. }
$$

Since in classical plate kinematics the term $u_{3}, \alpha$ corresponds to the rotation of the cross-section about the axis $x_{\alpha}$, the von Kàrmàn approximation can be interpreted as a theory retaining only moderate rotations and small strains [61].

\subsection{Refined LM4 model and Navier's solution}

The procedure for constructing the approximate $2 \mathrm{D}$ model is straightforward and is here just briefly recalled with reference to already published papers, e.g., [55,58,62]. Invoking variables' separation, the virtual and actual displacement and transverse stress variables are expanded along the thickness coordinate $z$ in each layer $k$ with a fourth-order polynomial:

$$
\begin{aligned}
\delta u_{i}^{k}\left(x_{\alpha}, z\right)=\sum_{\tau=0}^{4} F_{\tau}(z) \delta U_{i \tau}^{k}\left(x_{\alpha}\right) ; & u_{i}^{k}\left(x_{\alpha}, z\right)=\sum_{s=0}^{4} F_{s}(z) U_{i s}^{k}\left(x_{\alpha}\right) ; \\
\delta \sigma_{i 3}^{k}\left(x_{\alpha}, z\right)=\sum_{\tau=0}^{4} F_{\tau}(z) \delta T_{i 3 \tau}^{k}\left(x_{\alpha}\right) ; & \sigma_{i 3}^{k}\left(x_{\alpha}, z\right)=\sum_{s=0}^{4} F_{s}(z) T_{i 3 s}^{k}\left(x_{\alpha}\right) .
\end{aligned}
$$

The expansion along $z$ is defined in terms of Legendre's polynomials and can be found in the quoted references. The model in Eq. (15) is referred to as LM4, an acronym indicating that the model is a layer-wise mixed one and that it has an expansion of fourth order. This approximation can be enhanced upon subdividing each physical ply into several numerical layers. This may be of particular relevance for the short-wavelength response of the wrinkling instability, for which the rather thick core may be subdivided in a number of numerical layers $N_{l}^{\mathrm{c}}>1$. 
As far as the solution in the $2 \mathrm{D}$ domain $\Omega$ is concerned, the class of problems addressed in this work permits to find a closed-form solution according to Navier's method [61]. The in-plane distribution of the displacement and transverse stress variables is defined for each layer $k$ in terms of trigonometric functions, which exactly satisfy the simple support conditions at the plate boundaries:

$$
\begin{aligned}
& \left\{U_{1}^{k}\left(x_{\alpha}\right), T_{13}^{k}\left(x_{\alpha}\right)\right\}=\left\{\hat{U}_{1}^{k}, \hat{T}_{13}^{k}\right\}_{(m, n)} \cos \left(\frac{m \pi x_{1}}{a}\right) \sin \left(\frac{n \pi x_{2}}{b}\right) ; \\
& \left\{U_{2}^{k}\left(x_{\alpha}\right), T_{23}^{k}\left(x_{\alpha}\right)\right\}=\left\{\hat{U}_{2}^{k}, \hat{T}_{23}^{k}\right\}_{(m, n)} \sin \left(\frac{m \pi x_{1}}{a}\right) \cos \left(\frac{n \pi x_{2}}{b}\right) ; \\
& \left\{U_{3}^{k}\left(x_{\alpha}\right), T_{33}^{k}\left(x_{\alpha}\right)\right\}=\left\{\hat{U}_{3}^{k}, \hat{T}_{33}^{k}\right\}_{(m, n)} \sin \left(\frac{m \pi x_{1}}{a}\right) \sin \left(\frac{n \pi x_{2}}{b}\right) .
\end{aligned}
$$

The integer $m$ (resp. $n$ ) denotes the number of halfwaves of the periodic response along $x_{1}$ (resp. $x_{2}$ ) and defines, hence, its half-wavelength $L_{x}=a / m\left(\right.$ resp. $\left.L_{y}=b / n\right)$.

The assumed solution obtained from the combination of Eqs. (15) and (16) is substituted into the governing equation (13). Those terms whose virtual variation is differentiated with respect to the in-plane variables $x_{\alpha}$ are integrated-by-parts, which leads to the strong-form formulation of the $2 \mathrm{D}$ governing equations. Upon explicitly carrying out the derivations as well as the integrations over the thickness $h_{k}$ of each layer, the governing equation (13) can be written in terms of different $3 \times 3$ kernels, each of which is related to a couple of variables (virtual variation and actual unknown), a couple of $\tau, s$ (associated with the polynomial expansion along $z$ ) and to the $k$ th layer; the eigenvalue problem of Eq. (13) can thus be written as follows:

$$
\begin{array}{ll}
\delta \hat{\mathbf{U}}_{\tau}^{k}: & \mathbf{K}_{U U(m, n)}^{k \tau s} \hat{\mathbf{U}}_{s}^{k}+\mathbf{K}_{U T(m, n)}^{k \tau s} \hat{\mathbf{T}}_{s}^{k}+\lambda_{(m, n)} \mathbf{K}_{g(m, n)}^{k \tau s} \hat{\mathbf{U}}_{s}^{k}=\mathbf{0}, \\
\delta \hat{\mathbf{T}}_{\tau}^{k}: & \mathbf{K}_{T U(m, n)}^{k \tau s} \hat{\mathbf{U}}_{s}^{k}+\mathbf{K}_{T T(m, n)}^{k \tau s} \hat{\mathbf{T}}_{s}^{k}=\mathbf{0}
\end{array}
$$

where $\hat{\mathbf{U}}^{k}=\left[\begin{array}{lll}\hat{U}_{1}^{k} & \hat{U}_{2}^{k} & \hat{U}_{3}^{k}\end{array}\right]^{t}$ and $\hat{\mathbf{T}}^{k}=\left[\begin{array}{lll}\hat{T}_{13}^{k} & \hat{T}_{23}^{k} & \hat{T}_{33}^{k}\end{array}\right]^{t}$ are the vectors of unknown displacements and transverse stresses, respectively, and superscript $t$ denotes transposition. The kernels $\mathbf{K}_{U U(m, n)}^{k \tau s}, \mathbf{K}_{U T(m, n)}^{k \tau s}=$ $\left(\mathbf{K}_{T U(m, n)}^{k \tau s}\right)^{t}$ and $\mathbf{K}_{T T(m, n)}^{k \tau s}$ constitute the linear stiffness matrix related to the perturbed configuration. The kernel of the geometric stiffness matrix $\mathbf{K}_{g(m, n)}^{k \tau s}$ is constructed from the work done by the (virtual) nonlinear strains with the (actual) initial stress field $\sigma_{11}^{0 k}$; from the definition in Eq. (13.1), it is evident that this matrix depends on the initial stress field and is only related to the displacement unknowns. "Appendix" reports explicitly the terms constituting the kernels appearing in Eq. (17).

The $15 \times 15$ matrices for the $k$ th layer are obtained by expansion over the 5 terms $\tau, s=0,1, \ldots 4$. The construction of the arrays for the multilayered cross-section follows a standard assembly procedure that enforces exactly the continuity of displacements and transverse stresses between adjacent layers. The governing equation for the bifurcation buckling of the whole sandwich strut is finally given by the following classical eigenvalue problem:

$$
\left(\left[\begin{array}{ll}
\mathbf{K}_{U U(m, n)} & \mathbf{K}_{U T(m, n)} \\
\mathbf{K}_{U T(m, n)}^{t} & \mathbf{K}_{T T(m, n)}
\end{array}\right]+\lambda_{(m, n)}\left[\begin{array}{cc}
\mathbf{K}_{g(m, n)} & \mathbf{0} \\
\mathbf{0} & \mathbf{0}
\end{array}\right]\right)\left[\begin{array}{l}
\hat{\mathbf{U}}_{(m, n)} \\
\hat{\mathbf{T}}_{(m, n)}
\end{array}\right]=\left[\begin{array}{l}
\mathbf{0} \\
\mathbf{0}
\end{array}\right]
$$

\subsection{Solution of the eigenvalue problem}

Symmetric and antisymmetric modes The eigenvalue analysis of Eq. (18) provides for a given pair $(m, n)$ a number of eigenvalues that is equal to the number of degrees of freedom. Each eigenvalue corresponds thus to a mode that is characterized by (a) the half-wavelengths $L_{x}=a / m, L_{y}=b / n$ in the plane $\Omega$, and $(b)$ by the modal shapes of the displacements and transverse stresses across the thickness, which is given by the eigenvector. Throughout this article, we will conventionally refer to a global mode whenever $L_{x}=a$ (i.e., $m=1$ ) and to a local mode (wrinkling) whenever the half-wavelength along the $x_{1}$ direction is shorter than the panel's dimension (i.e., $m>1$ ). Inspection of the through-thickness modal distributions permits to identify symmetric and antisymmetric modes according to the following definitions: 
Antisymmetric modes: The transverse deflection $u_{3}$ is an even function of the thickness coordinate $z$ while the in-plane displacements $u_{\alpha}(z)$ are odd functions:

$$
u_{3}\left(x_{\alpha},-z\right)=u_{3}\left(x_{\alpha}, z\right) ; \quad u_{\alpha}\left(x_{\alpha},-z\right)=-u_{\alpha}\left(x_{\alpha}, z\right) .
$$

Global buckling modes are always antisymmetric since the perturbed configuration is characterized by a transverse deflection of the reference surface.

Symmetric modes: The transverse displacement $u_{3}(z)$ is an odd function, while in-plane displacements $u_{\alpha}(z)$ are even functions:

$$
u_{3}\left(x_{\alpha},-z\right)=-u_{3}\left(x_{\alpha}, z\right) ; \quad u_{\alpha}\left(x_{\alpha},-z\right)=u_{\alpha}\left(x_{\alpha}, z\right) .
$$

Since the mid-surface of the sandwich panel does not experience any deflection, the symmetric mode is only a local buckling mode (wrinkling).

Critical loads and buckling loads For a given half-wavelength $L_{x}=a / m, L_{y}=b / n$, each eigenvalue $\lambda_{(m, n)}$ corresponds to a critical load $N_{\mathrm{cr}}$ that is defined for a prescribed initial stress acting in the face sheets as

$$
N_{\mathrm{cr}}^{\sigma}=2 h_{\mathrm{f}}\left(\lambda_{(m, n)} \sigma_{11}^{0}\right),
$$

or for a uniformly strained cross-section as

$$
N_{\mathrm{cr}}^{\epsilon}=\sum_{k=1}^{N_{l}} \int_{h_{k}} C_{11}^{k} \mathrm{~d} z\left(\lambda_{(m, n)} \epsilon_{11}^{0}\right)=\lambda_{(m, n)} A_{11} \epsilon_{11}^{0} .
$$

For simplicity, the preload is a unit compressive stretch or a unit compressive stress.

The lowest critical load obtained for all possible pairs $(m, n)$ defines the buckling load of the plate, which will be indicated by $P^{\sigma}$ or $P^{\epsilon}$ depending on the definition of the initial stress state:

$$
\left\{P^{\sigma}, P^{\epsilon}\right\}=\min _{m, n}\left\{N_{\mathrm{cr}}^{\sigma}, N_{\mathrm{cr}}^{\epsilon}\right\}
$$

The buckling wavelength is thus defined by the pair $(m, n)$ that is associated with the buckling load.

\section{Numerical results and discussion}

This section presents extensive numerical results for global and local buckling of sandwich panels. All sandwich cross-sections are symmetric and subjected to uniaxial compression along the longitudinal $x_{1}$ direction. The case studies discussed in Sects. 3.1-3.4 refer to wide sandwich struts: These are analyzed in a plane strain setting in the $x_{1} z$ plane, which is imposed within the present solution by prescribing $n=0$. The case study discussed in Sect. 3.5 concerns the uniaxial compression of a square sandwich plate with simply supported edges: in this case $n=1$ is set. Cellular materials (e.g., honeycomb cores) are represented as equivalent continua characterized by homogenized elastic properties.

\subsection{Convergence analysis}

A preliminary analysis is performed to establish the convergence behavior of the present LM4 model. In particular, we shall investigate the effect of the number of computational layers $N_{l}^{\mathrm{c}}$ employed for the core. Convergence with respect to $N_{l}^{\mathrm{c}}$ is considered for both the buckling load and the instability mode, which is identified by the number of halfwaves $m$ and by specifying whether the mode is symmetric (S) or antisymmetric (A). The wide strut (plane strain in $x_{1} z$ plane) has a total thickness of $h$ and a length $a=5 h$. The face sheets are isotropic with Young's modulus $E_{\mathrm{f}}$. The core's moduli are parametrically defined as follows: $E_{3}^{\mathrm{c}}=k E_{\mathrm{f}}$, $E_{1}^{\mathrm{c}}=E_{2}^{\mathrm{c}}=\chi E_{3}^{\mathrm{c}}, G_{13}^{\mathrm{c}}=G_{23}^{\mathrm{c}}=0.5 E_{3}^{\mathrm{c}}, G_{12}^{\mathrm{c}}=E_{1}^{\mathrm{c}} /\left(2\left(1+v_{12}^{\mathrm{c}}\right)\right)$. The initial stress field is prescribed by uniformly straining the whole cross-section. Several face thickness ratios $R_{\mathrm{f}}=h_{\mathrm{f}} / h$, face-to-core stiffness ratios $k=E_{3}^{\mathrm{c}} / E_{\mathrm{f}}$ and core's orthotropic ratios $\chi=E_{1}^{\mathrm{c}} / E_{3}^{\mathrm{c}}$ are considered. These parameters are known to influence the buckling wavelength and may hence affect the accuracy of the LM4 model. Comparison is made with respect to converged FEM results obtained with the commercial software packages Abaqus and Ansys. 
Table 1 Convergence of buckling loads $P^{\epsilon}(\mathrm{N} / \mathrm{mm})$ of LM4 models with different number of mathematical layers used in the core $\left(N_{l}^{\mathrm{c}}\right)$ for different sandwich configurations

\begin{tabular}{|c|c|c|c|c|c|c|}
\hline \multirow[t]{2}{*}{$N_{l}^{\mathrm{c}}$} & \multicolumn{2}{|c|}{$\begin{array}{l}R_{\mathrm{f}} \\
(k=0.002, \chi=1)\end{array}$} & \multicolumn{2}{|c|}{$\stackrel{k}{\left(R_{\mathrm{f}}=0.02, \chi=1\right)}$} & \multicolumn{2}{|c|}{$\begin{array}{l}\chi \\
\left(R_{\mathrm{f}}=0.02, k=0.02\right)\end{array}$} \\
\hline & 0.01 & 0.1 & $10^{-3}$ & $10^{-1}$ & $10^{-3}$ & $10^{-1}$ \\
\hline 1 & $\begin{array}{l}838.99 \\
(31, \mathrm{~A})\end{array}$ & $\begin{array}{l}3,528.0 \\
(1, A)\end{array}$ & $\begin{array}{l}948.46 \\
(12, \mathrm{~A})\end{array}$ & $\begin{array}{l}19,791 \\
(1, A)\end{array}$ & $\begin{array}{l}2,758.0 \\
(24, S)\end{array}$ & $\begin{array}{l}6,688.9 \\
(30, S)\end{array}$ \\
\hline 2 & $\begin{array}{l}816.81 \\
(32, \mathrm{~A} / \mathrm{S})\end{array}$ & $\begin{array}{l}3,528.0 \\
(1, \mathrm{~A})\end{array}$ & $\begin{array}{l}945.13 \\
(13, A)\end{array}$ & $\begin{array}{l}19,791 \\
(1, \mathrm{~A})\end{array}$ & $\begin{array}{l}2,758.0 \\
(24, S)\end{array}$ & $\begin{array}{l}6,435.0 \\
(33, S)\end{array}$ \\
\hline 3 & $\begin{array}{l}813.47 \\
(32, \mathrm{~A} / \mathrm{S})\end{array}$ & $\begin{array}{l}3,528.0 \\
(1, \mathrm{~A})\end{array}$ & $\begin{array}{l}945.02 \\
(13, \mathrm{~A})\end{array}$ & $\begin{array}{l}19,791 \\
(1, \mathrm{~A})\end{array}$ & $\begin{array}{l}2,758.0 \\
(24, S)\end{array}$ & $\begin{array}{l}6,426.9 \\
(33, \mathrm{~A} / \mathrm{S})\end{array}$ \\
\hline 5 & $\begin{array}{l}812.78 \\
(32, \mathrm{~A} / \mathrm{S})\end{array}$ & $\begin{array}{l}3,528.0 \\
(1, \mathrm{~A})\end{array}$ & $\begin{array}{l}945.02 \\
(13, \mathrm{~A})\end{array}$ & $\begin{array}{l}19,791 \\
(1, \mathrm{~A})\end{array}$ & $\begin{array}{l}2,758.0 \\
(24, S)\end{array}$ & $\begin{array}{l}6,426.0 \\
(33, \mathrm{~A} / \mathrm{S})\end{array}$ \\
\hline 10 & $\begin{array}{l}812.72 \\
(32, \mathrm{~A} / \mathrm{S})\end{array}$ & $\begin{array}{l}3,528.0 \\
(1, \mathrm{~A})\end{array}$ & $\begin{array}{l}945.02 \\
(13, \mathrm{~A})\end{array}$ & $\begin{array}{l}19,791 \\
(1, \mathrm{~A})\end{array}$ & $\begin{array}{l}2,758.0 \\
(24, S)\end{array}$ & $\begin{array}{l}6,425.9 \\
(33, \mathrm{~A} / \mathrm{S})\end{array}$ \\
\hline Ansys & $\begin{array}{l}818.8 \\
(31, \mathrm{~A} / \mathrm{S})\end{array}$ & $\begin{array}{l}3,528.0 \\
(1, \mathrm{~A})\end{array}$ & $\begin{array}{l}945.93 \\
(13, \mathrm{~A})\end{array}$ & $\begin{array}{l}19,792 \\
(1, \mathrm{~A})\end{array}$ & $\begin{array}{l}2,767.6 \\
(23, S)\end{array}$ & $\begin{array}{l}6,464.9 \\
(33, \mathrm{~A} / \mathrm{S})\end{array}$ \\
\hline Abaqus & $\begin{array}{l}807.85 \\
(31, \mathrm{~A} / \mathrm{S})\end{array}$ & $\begin{array}{l}3,533.4 \\
(1, \mathrm{~A})\end{array}$ & $\begin{array}{l}950.95 \\
(13, \mathrm{~A})\end{array}$ & $\begin{array}{l}21,468 \\
(1, \mathrm{~A})\end{array}$ & $\begin{array}{l}2,752.4 \\
(23, \mathrm{~S})\end{array}$ & $\begin{array}{l}6,546.0 \\
(33, \mathrm{~A} / \mathrm{S}) \\
\end{array}$ \\
\hline
\end{tabular}

The parentheses identify the buckling mode: number of halfwaves $m$ and modal shape (antisymmetric or symmetric). Entries in bold denote converged solutions

All FE models employ linear (four node) plane strain elements. The FE mesh consists of 256 elements along the $x_{1}$ axis for both face sheets and the core, while along the thickness 60 elements are used for the core and 4 elements are used for each face sheet. For the numerical evaluation, $h=50 \mathrm{~mm}$ and $E_{\mathrm{f}}=70 \mathrm{GPa}$ have been used; moreover, all Poisson's ratios are set to zero to facilitate the imposition of a uniaxial initial stress $\sigma_{11}^{0 k}$ in FEM solutions.

The results are listed in Table 1, where entries in bold indicate converged solutions, i.e., solutions providing the correct mode and with errors in the buckling load that are less than $0.5 \%$. In many cases, critical loads corresponding to symmetric and antisymmetric modes are practically coincident: The label A/S has been thus used whenever the difference between these loads is less than $0.05 \%$. Convergence of the proposed approach is clearly demonstrated. Moreover, an excellent agreement is found with respect to FEM results, in particular those provided by Ansys. The somewhat larger discrepancies of Abaqus results are attributed to the inconsistent stress measure employed in this software, as reported in [51]. In general, one computational layer for the core is sufficient to determine accurately the overall buckling behavior and to identify the occurrence of a short-wavelength wrinkling response. Depending on the configurations, several numerical layers $N_{l}^{\mathrm{c}}$ may be necessary in order to precisely determine the wrinkling mode. For all the considered cases, $N_{l}^{\mathrm{c}}=3$ ensures a converged solution.

\subsection{Global buckling}

The overall (single halfwave) buckling of a wide sandwich strut $(m=1, n=0)$ is considered by referring to the elasticity solution proposed by Kardomateas [28]. The strut has a slenderness ratio $a / h=30(h=30 \mathrm{~mm}$ has been taken for the numerical evaluations). The face sheets are made of unidirectional carbon or glass fiber reinforced plastics (CFRP or GFRP); the core may be an orthotropic honeycomb or an isotropic foam; the corresponding material data are reported in Table 2 . The initial load is prescribed by a uniform axial strain $\varepsilon_{11}^{0}$ as in [28]. Based on the convergence results in Table 1, only one numerical layer will be employed for the core $\left(N_{l}^{\mathrm{c}}=1\right)$.

Non-dimensional overall buckling loads $P^{\epsilon} / P_{\mathrm{E}}$, where $P_{\mathrm{E}}$ is Euler's load for a beam given in Eq. (1), are reported in Table 3 for a CFRP/honeycomb sandwich with thickness ratios $R_{\mathrm{f}}=h_{\mathrm{f}} / h$ varying in the range considered in [28]. It is seen that $P^{\epsilon}$ of the present LM4 model recovers exactly the elasticity solution. No substantial difference occurs if von Kàrmàn approximation is used, see $P_{v K}^{\epsilon}$, nor if the initial load is applied by a uniform stress acting in the face sheets (not shown for conciseness). The behavior of the present LM4 solution, the elasticity solution of Kardomateas as well the approximated formulas proposed by Allen, denoted $P_{\mathrm{A} 1}$ and $P_{\mathrm{A} 2}$ and defined in Eqs. (2) and (3), respectively, are graphically compared in Fig. 3. Allen's load for 
Table 2 Material data for the overall buckling analysis

\begin{tabular}{|c|c|c|c|c|}
\hline & \multicolumn{2}{|l|}{ Skin } & \multicolumn{2}{|l|}{ Core } \\
\hline & CFRP & GFRP & Honeycomb & PVC foam \\
\hline$E_{1}(\mathrm{MPa})$ & $181 \times 10^{3}$ & $40 \times 10^{3}$ & 32.0 & 100.0 \\
\hline$E_{2}(\mathrm{MPa})$ & $10.3 \times 10^{3}$ & $10 \times 10^{3}$ & 32.0 & 100.0 \\
\hline$E_{3}(\mathrm{MPa})$ & $10.3 \times 10^{3}$ & $10 \times 10^{3}$ & 300.0 & 100.0 \\
\hline$G_{23}(\mathrm{MPa})$ & $5.96 \times 10^{3}$ & $3.5 \times 10^{3}$ & 48.0 & 38.46 \\
\hline$G_{13}(\mathrm{MPa})$ & $7.17 \times 10^{3}$ & $4.5 \times 10^{3}$ & 48.0 & 38.46 \\
\hline$G_{12}(\mathrm{MPa})$ & $7.17 \times 10^{3}$ & $4.5 \times 10^{3}$ & 13.0 & 38.46 \\
\hline$v_{23}(-)$ & 0.400 & 0.400 & 0.25 & 0.30 \\
\hline$v_{13}(-)$ & 0.277 & 0.26 & 0.25 & 0.30 \\
\hline$v_{12}(-)$ & 0.277 & 0.065 & 0.25 & 0.30 \\
\hline
\end{tabular}

Table 3 Non-dimensional overall buckling loads $P_{\mathrm{cr}} / P_{\mathrm{E}}$ for the CFRP/honeycomb sandwich

\begin{tabular}{lllllll}
\hline$h_{\mathrm{f}} / h$ & 0.02 & 0.04 & 0.08 & 0.12 & 0.16 & 0.20 \\
\hline Elasticity [28] & 0.7173 & 0.5692 & 0.4205 & 0.3508 & 0.3161 & 0.3018 \\
$P^{\epsilon}$ & 0.7172 & 0.5691 & 0.4205 & 0.3508 & 0.3161 & 0.3018 \\
$P_{v K}^{\epsilon}$ & 0.7182 & 0.5696 & 0.4207 & 0.3509 & 0.3162 & 0.3018 \\
\hline
\end{tabular}

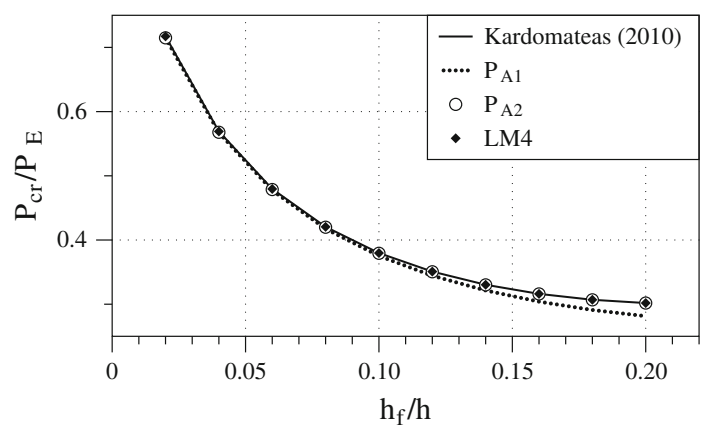

Fig. 3 Non-dimensional overall buckling loads $P_{\mathrm{cr}} / P_{\mathrm{E}}$ for different face sheet thickness ratios $R_{\mathrm{f}}=h_{\mathrm{f}} / h(\mathrm{CFRP} / \mathrm{honeycomb}$ sandwich)

thin face sheets $P_{\mathrm{A} 1}$ slightly deviates from the elasticity solution as the thickness of the face sheets increases, whereas very good agreement is found with Allen's formula for thick face sheets $P_{\mathrm{A} 2}$ over the whole range of $R_{\mathrm{f}}$ ratios. The modal distributions through the sandwich cross-section of the axial displacement $u_{1}(z)$ and the transverse displacement $u_{3}(z)$ as well as of the transverse stresses $\sigma_{13}(z)$ and $\sigma_{33}(z)$ are displayed in Fig. 4 for $R_{\mathrm{f}}=0.04$ and $R_{\mathrm{f}}=0.2$. These through-thickness distributions are the amplitudes of the periodic response in $x_{\alpha}$ described in Eq. (16). Displacement and transverse stress modes have been normalized with respect to the maximum value of the transverse displacement $w_{\max }=u_{3}(z=0)$ and of the transverse shear stress $\sigma_{13 \max }=\sigma_{13}(z=0)$, respectively. Note that the present LM4 model is capable of capturing the characteristic gradients at the bi-material skin-core interfaces.

Non-dimensional overall buckling loads $P_{\mathrm{cr}} / P_{\mathrm{E}}$ are reported in Fig. 5 for a CFRP/foam sandwich with varying $E_{1}^{\mathrm{f}} / E_{\mathrm{c}}$ ratios and in Fig. 6 for a GFRP/foam sandwich with varying $a / h$ ratios. In both cases, rather thick face sheets have been considered with $R_{\mathrm{f}}=0.1$. Comparison with the elasticity solution, whose data have been digitally extracted from the graphs reported in [28], shows again a perfect agreement of the present quasi-3D LM4 model. Results referring to von Kàrmàn approximation $\left(P_{v K}^{\epsilon}\right)$ and to a uniform stress preload $\left(P_{v K}^{\sigma}\right)$ have not been included in the figures because they practically coincide with the loads $P^{\epsilon}$.

Note that Fig. 6 reports global buckling loads of very short struts $(a / h<0.5)$, which permits to highlight a substantial difference between Allen's analytical formulas for $P_{\mathrm{A} 1}$ (thin face sheets) and $P_{\mathrm{A} 2}$ (thick face sheets). As the slenderness tends to zero $(a / h \rightarrow 0)$, the bending stiffness and, hence, Euler's load $P_{\mathrm{E}}$ increase to infinity. According to Engesser's theory, Allen's buckling load $P_{\mathrm{A} 1}$ tends to the finite value of the shear crimping load $P_{\mathrm{c}}$, and the non-dimensional result $P_{\mathrm{A} 1} / P_{\mathrm{E}}$ does thus tend toward zero. On the contrary, the non-dimensional Allen's load $P_{\mathrm{A} 2} / P_{\mathrm{E}}$ is shown to tend to the finite value corresponding to $P_{\mathrm{Ef}} / P_{\mathrm{E}}$, where 

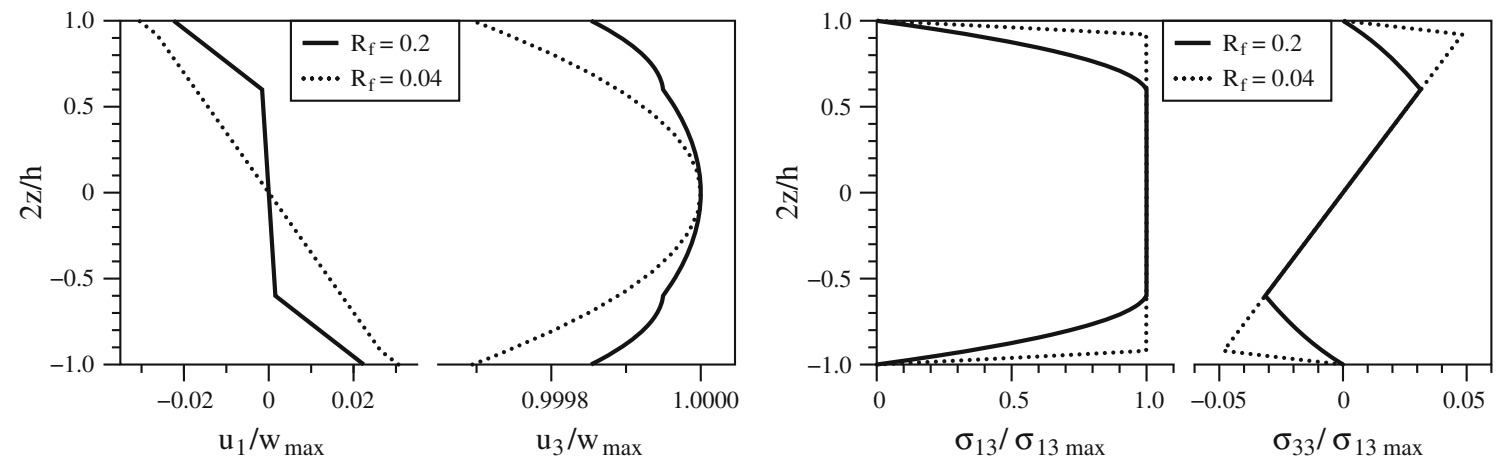

Fig. 4 Normalized through-thickness modal shapes for the global buckling of the wide CFRP/honeycomb sandwich strut with thin $\left(R_{\mathrm{f}}=0.04\right)$ and thick $\left(R_{\mathrm{f}}=0.2\right)$ face sheets: displacement modes $(l e f t)$ and transverse stress modes $(r i g h t)$

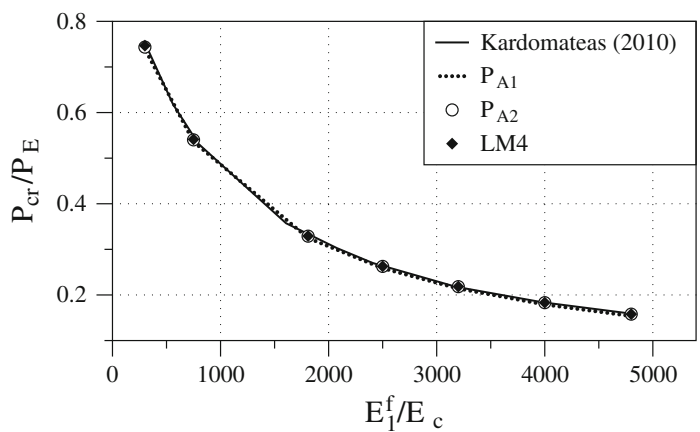

Fig. 5 Non-dimensional global buckling loads $P_{\mathrm{cr}} / P_{\mathrm{E}}$ for different stiffness ratios $E_{1}^{\mathrm{f}} / E_{\mathrm{c}}$ (wide CFRP/foam sandwich strut with $a / h=30$ and $\left.R_{\mathrm{f}}=0.1\right)$

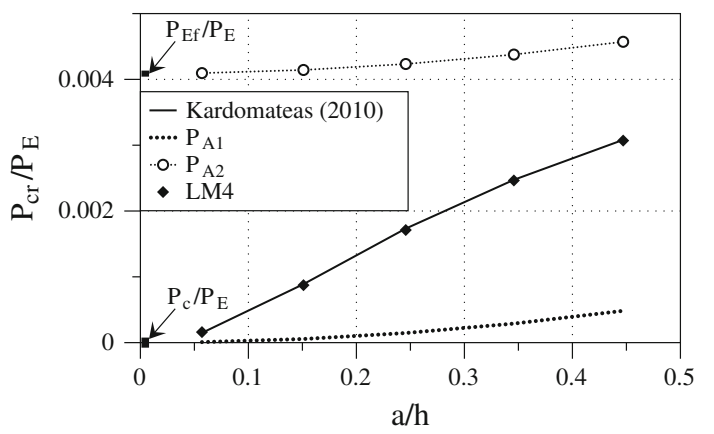

Fig. 6 Non-dimensional global buckling loads $P_{\mathrm{cr}} / P_{\mathrm{E}}$ of wide struts for varying slenderness $a / h$ : asymptotic behavior of present LM4 model and Allen's loads $P_{\mathrm{A} 1}$ and $P_{\mathrm{A} 2}$ for very thick struts (GFRP/foam sandwich with $R_{\mathrm{f}}=0.1$ )

$P_{\mathrm{Ef}}$ is the critical load of the face sheets when they behave as independent struts, see Eq. (3.2). Results of the present model exactly match the 3D solution.

\subsection{Buckling of sandwich struts with isotropic skins and core}

Overall, long wavelength buckling is the dominant failure mechanism of slender struts. If the strut is rather short and the face-to-core thickness ratio $h_{\mathrm{f}} / h_{\mathrm{c}}$ is sufficiently small, the short-wavelength wrinkling instability may appear. Moreover, the instability wavelength is known to depend on the stiffness ratio between face sheet and core materials. In order to consider the transition between these two instability modes, the buckling behavior is analyzed for different geometrical ratios $R_{\mathrm{f}}=h_{\mathrm{f}} / h$ and $a / h$ as well as for different stiffness ratios $E_{\mathrm{c}} / E_{\mathrm{f}}$. Both skins and core materials are taken to be isotropic, and the following material parameters are used unless 


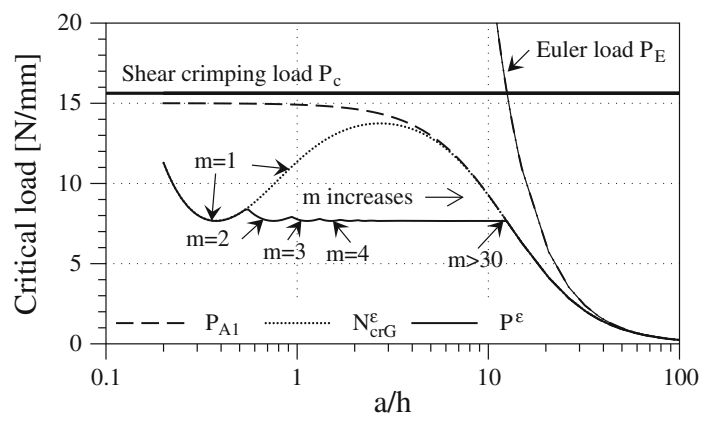

Fig. 7 Evolution of buckling modes of a wide sandwich strut for varying slenderness ratio $a / h$
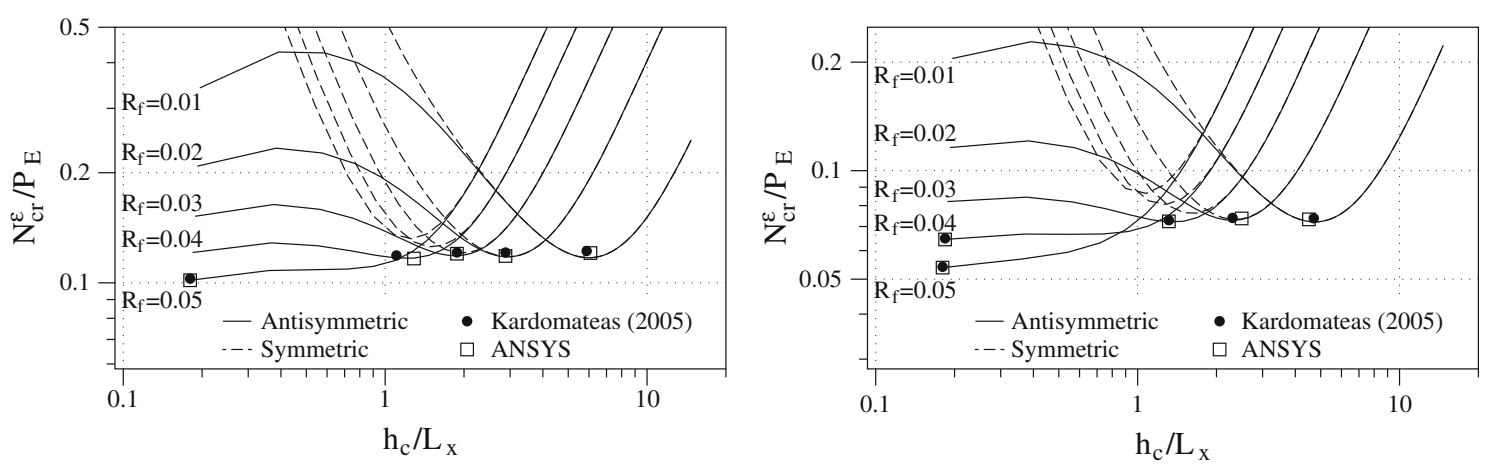

Fig. 8 Non-dimensional critical loads $N_{\mathrm{cr}}^{\epsilon} / P_{\mathrm{E}}$ versus ratio of core thickness to half-wavelength $h_{\mathrm{c}} / L_{x}=m\left(h_{\mathrm{c}} / a\right)$ for different face-to-core thickness ratios $R_{\mathrm{f}}=h_{\mathrm{f}} / h$ and two stiffness ratios: $E_{\mathrm{f}} / E_{\mathrm{c}}=500($ left $)$ and $E_{\mathrm{f}} / E_{\mathrm{c}}=1,000$ (right). The abscissa for the global buckling $(m=1)$ is $h_{\mathrm{c}} / a=0.196$

otherwise stated: $E_{\mathrm{c}}=1 \mathrm{MPa}, v_{\mathrm{c}}=0, E_{\mathrm{f}}$ variable, $v_{\mathrm{f}}=0.35$. Furthermore, $N_{l}^{\mathrm{c}}=1$ will be used for all LM4 results by keeping in mind the results of the convergence analysis of Table 1.

The evolution of the buckling load with the slenderness $a / h$ of the sandwich strut is displayed in Fig. 7 ( $h=30 \mathrm{~mm}$ has been chosen for the numerical evaluations). The strut has rather thin face sheets with $R_{\mathrm{f}}=h_{\mathrm{f}} / h=0.02$ and a face-to-core stiffness ratio of $E_{\mathrm{f}} / E_{\mathrm{c}}=750$, which is a typical ratio for sandwich panels. Figure 7 reports the critical global buckling loads (i.e., the critical load obtained with $m=1$ ), denoted $N_{\mathrm{cr} G}^{\epsilon}$, and the buckling load $P^{\epsilon}$, which may be associated with a higher number of halfwaves $m$. For comparison purposes, Euler's load $P_{\mathrm{E}}$ as given by Eq. (1) and Allen's load $P_{\mathrm{A} 1}$ for thin face sheets, given in Eq. (2), are displayed as well. Euler's formula provides satisfactory results only for large slenderness ratios $a / h$. Transverse shear effects, as accounted for by Allen's formula $P_{\mathrm{A} 1}$ and the present model, begin to play a relevant role for shorter struts with $a / h<30$ by substantially reducing the critical load. At $a / h \approx 12$, the present LM4 model predicts an abrupt change in the buckling behavior: The buckling mode is no longer a global one with $m=1$, but switches to a local one characterized by a higher number of halfwaves $m$. For the considered configuration, the switch between global and local instability occurs with $m>30$ with a half-wavelength $L_{x}=a / m \approx 11 \mathrm{~mm}$, which is approximately one third of the thickness of the core. Therefore, this local instability may be classified as wrinkling. Comparison with the shear crimping load $P_{\mathrm{c}}$ defined in Eq. (2.3) shows that wrinkling is expected to occur at a lower load than the core shear failure. It is emphasized that the present model cannot actually represent the shear crimping because this failure mode is an aperiodic response.

Figure 8 reports non-dimensional critical loads $N_{\mathrm{cr}}^{\epsilon} / P_{\mathrm{E}}$ versus the ratio $h_{\mathrm{c}} / L_{x}=m\left(h_{\mathrm{c}} / a\right)$ for various configurations of the wide sandwich strut. In these graphs, the global buckling with $m=1$ is identified by the smallest value of the abscissa $h_{\mathrm{c}} / L_{x}=h_{\mathrm{c}} / a$. The evolution of the critical loads associated with both the symmetric and the antisymmetric modes is displayed in the figure: for the present case of isotropic core materials, antisymmetric wrinkling is shown to dominate with respect to symmetric wrinkling, which agrees with precedent analyses, e.g., [26,29]. Furthermore, comparison with the elasticity solution of Kardomateas [49] as well as with the FEM solution obtained by Ansys shows a good quantitative agreement. For "thick" face sheets $\left(R_{\mathrm{f}}=0.05\right)$, global buckling occurs at lower loads than wrinkling. For thin face sheets $\left(R_{\mathrm{f}}=0.01\right)$, wrinkling dominates with half-wavelengths $L_{x}$ that are shorter than the core thickness $h_{\mathrm{c}}$. For a given stiffness ratio 

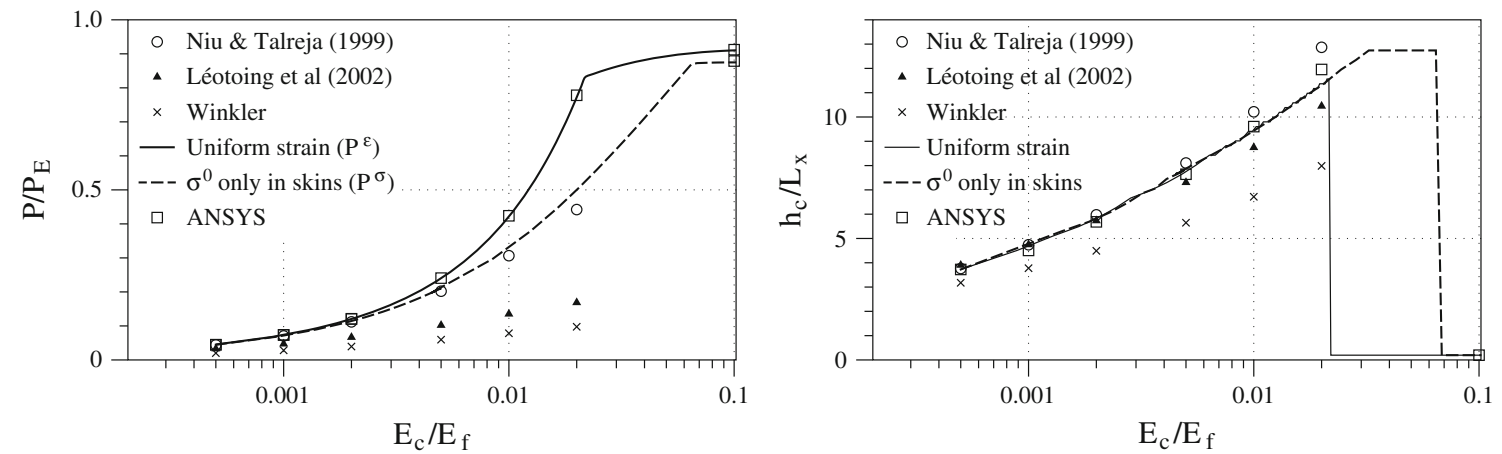

Fig. 9 Global buckling and wrinkling behavior predicted by different models for variable core-to-face stiffness ratio $E_{\mathrm{c}} / E_{\mathrm{f}}$ : non-dimensional buckling loads $P / P_{\mathrm{E}}(l e f t)$ and normalized buckling half-wavelength $h_{\mathrm{c}} / L_{x}($ right $)\left(R_{\mathrm{f}}=0.01, a / h=5\right)$

$E_{\mathrm{f}} / E_{\mathrm{c}}$, the thicker is the core the shorter is the buckling wavelength. The buckling wavelength is shown to depend on the stiffness ratio $E_{\mathrm{f}} / E_{\mathrm{c}}$ as well: for $R_{\mathrm{f}}=0.04$, the core is not "sufficiently thick" to allow wrinkling if $E_{\mathrm{f}} / E_{\mathrm{c}}=1000$, whereas for $E_{\mathrm{f}} / E_{\mathrm{c}}=500$ the "thickness" of the core allows a wrinkling instability to occur before the overall instability. In addition, the critical loads for symmetric and antisymmetric modes are shown to coincide for sufficiently short wavelengths, which occurs when the core is sufficiently thick to prevent the interaction between the top and bottom face sheets. The present LM4 model recovers thus the dependence of this interaction on the geometrical and constitutive properties of the sandwich strut, with no need to refer to a decay model. FEM results confirm this behavior since for short wavelengths the wrinkling loads of antisymmetric and symmetric mode coincide, while in general the antisymmetric mode provides the lowest wrinkling load.

Figure 9 reports the buckling loads and the corresponding half-wavelength ratio $h_{\mathrm{c}} / L_{x}=m\left(h_{\mathrm{c}} / a\right)$ obtained by different formulations for varying core-to-face stiffness ratio $E_{\mathrm{c}} / E_{\mathrm{f}}$. The geometry of the sandwich strut is defined by the ratios $R_{\mathrm{f}}=0.01$ and $a / h=5$. Present results obtained by a uniform initial strain prescribed over the whole cross-section ( $P^{\epsilon}$ formulation) and by an initial stress $\sigma_{11}^{0}$ prescribed only in the face sheets ( $P^{\sigma}$ formulation) are compared with several analytical wrinkling models available in the literature and FE results by Ansys (2D plane strain elements, mesh given in Sect. 3.1). FEM results obtained when the core carries the initial axial load are in excellent agreement with the present $P^{\epsilon}$ formulation. The FE analysis for the case in which the core is free from the axial preload predicts an aperiodic response, where the out-of-plane deflection is localized at the loaded edges (edge buckling), see also [50]. These results are not reported in Fig. 9 because the present analysis is only concerned with a periodic response. All wrinkling models taken from the literature assume the initial load entirely carried by the face sheets and should, therefore, be rather compared with the present $P^{\sigma}$ formulation. For low core stiffness, all formulations are in a rather good agreement: The wrinkling load increases and the wrinkling wavelength decreases as the core gets stiffer. For the considered configuration, half-wavelengths of less than $1 / 10$ of the core thickness are obtained for $E_{\mathrm{c}}>0.01 E_{\mathrm{f}}$. The differences between the various models appear as the core stiffness increases. An excellent agreement is found with Niu and Talreja's model [29], in which the core is modeled as an isotropic continuum (i.e., the axial stiffness of the core is retained). Some differences are visible when $E_{\mathrm{c}}>0.01 E_{\mathrm{f}}$, where Niu and Talreja's model predicts lower critical loads with respect to the present $P^{\sigma}$ model. The discrepancy is attributed to the Euler-Bernoulli beam theory employed in Niu and Talreja's model of the face sheets. In fact, for $E_{\mathrm{c}}>0.01 E_{\mathrm{f}}$ the half-wavelength decreases such that $h_{\mathrm{c}} / L_{x}>10$ and, since $R_{\mathrm{f}}=h_{\mathrm{f}} / h_{\mathrm{c}}=0.01$, the length-to-thickness ratio of the bent face sheet decreases to $L_{x} / h_{\mathrm{f}}<10$, which is an aspect ratio for which the "thin beam" assumptions for the face sheets may lead to some inaccuracies. Larger discrepancies are found for the model of Lèotoing et al. [43], that discards the axial stiffness of the core (anti-plane core) and yields clearly lower buckling loads. The simplest wrinkling model based on Winkler's elastic foundation model additionally discards the transverse shear deformability of the core and produces, hence, buckling loads that are even lower. Allen [26] stated that Winkler's approximation is actually valid only for large wavelengths, say $h_{\mathrm{c}} / L_{x}<3$, a result that is confirmed by the present investigation. Finally, if the $E_{\mathrm{c}} / E_{\mathrm{f}}$ ratio is too high, the characteristic sandwich heterogeneity is weakened and global buckling dominates instead of wrinkling. Since the global buckling load is barely affected by the preload formulation (see Sect. 3.2), the transition from local to global instability occurs at this global buckling load, which corresponds to different values of $E_{\mathrm{c}} / E_{\mathrm{f}}$ depending on whether the core carries the axial preload or not. FEM predictions for global buckling loads do perfectly agree with present $P^{\epsilon}$ and $P^{\sigma}$ formulations. 


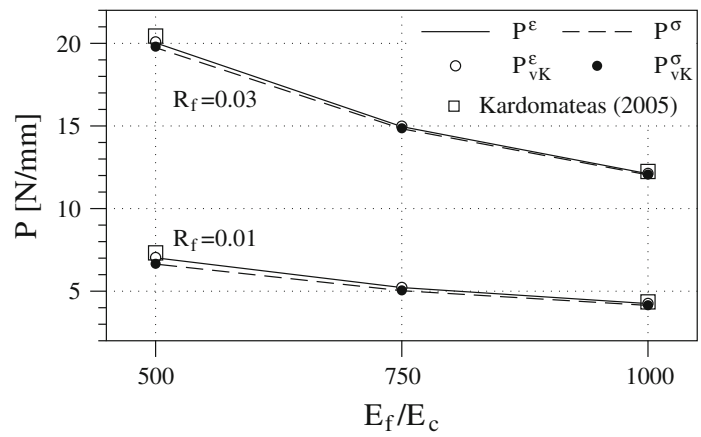

Fig. 10 Wrinkling loads $P$ (per unit width) for three characteristic stiffness ratios $E_{\mathrm{f}} / E_{\mathrm{c}}$ and two face sheet thickness ratios $R_{\mathrm{f}}$ : assessment of four formulations
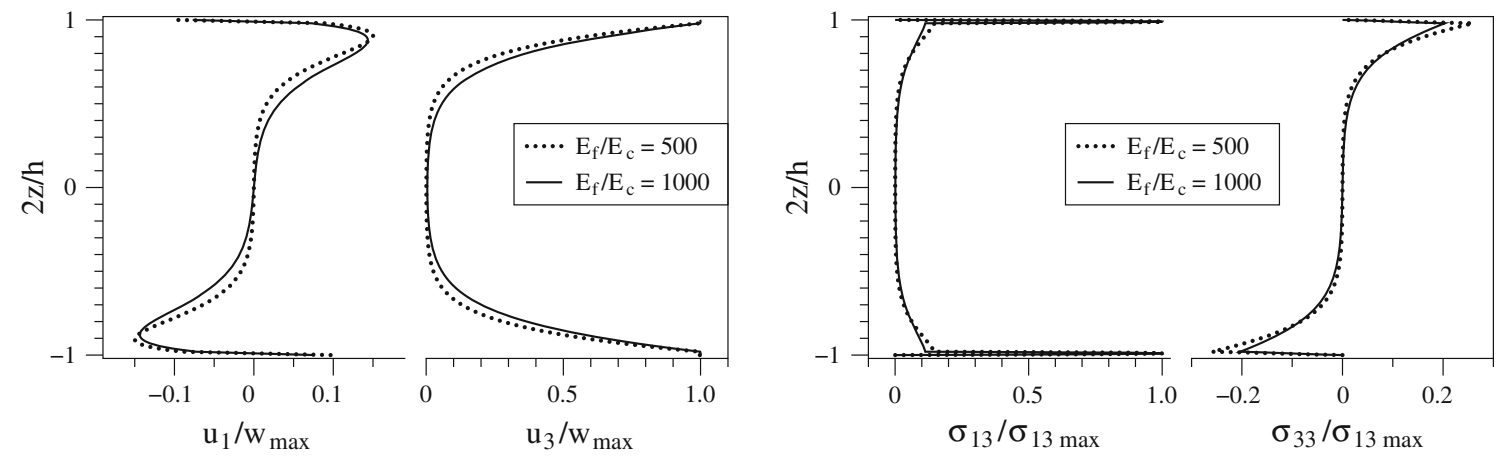

Fig. 11 Antisymmetric wrinkling modes of a sandwich strut with isotropic face sheets and core $\left(R_{\mathrm{f}}=0.01\right)$ for two stiffness ratios $E_{\mathrm{f}} / E_{\mathrm{c}}$ : through-thickness modal shapes of the axial and transverse displacements $u_{1}$ and $u_{3}$ (left) and of the transverse shear and normal stresses $\sigma_{13}$ and $\sigma_{33}$ (right)

The role of von Kàrmàn's nonlinearities is assessed in Fig. 10, where wrinkling loads per unit width are reported for $E_{\mathrm{f}} / E_{\mathrm{c}}$ ratios between 500 and 1,000, a usual range for sandwich structures, and for two face sheet thickness ratios $\left(R_{\mathrm{f}}=0.01\right.$ and 0.03$)$. A short strut with $a / h=5$ has been considered, with $E_{\mathrm{f}}=1 \mathrm{GPa}, v_{\mathrm{f}}=0.35$ and $v_{\mathrm{c}}=0$. Only marginal differences are found between the different models: Very small differences can be observed between the initial load formulations only for the stiffest core $\left(E_{\mathrm{f}} / E_{\mathrm{c}}=500\right)$, while von Kàrmàn's nonlinearities appear to have no effects irrespective of the sandwich configurations. The excellent agreement with the elasticity solution of Kardomateas [49] is confirmed.

In closure of this section, the shapes of the antisymmetric and symmetric wrinkling modes are illustrated in order to highlight the quasi-3D accuracy of the present LM4 model. Through-thickness modal shapes for a sandwich strut with thin faces $\left(R_{\mathrm{f}}=0.01\right)$ are plotted in Fig. 11 for the antisymmetric wrinkling mode and in Fig. 12 for the symmetric one. The figures highlight the exact verification of the interlaminar continuity of displacements and transverse stresses. Very steep transverse stress gradients arise inside the thin face sheets, where the maximum transverse shear stress $\sigma_{13 \text { max }}$ occurs at the mid-plane of the face: This aspect cannot be considered if Euler-Bernoulli's theory is used for the face sheets.

\subsection{Wrinkling of sandwich struts with orthotropic core}

This section discusses the buckling behavior of sandwich struts with cores having a transverse modulus $E_{3}^{\mathrm{c}}$ that is higher than the axial one $E_{1}^{\mathrm{c}}$. This case is representative for cellular cores like honeycombs. A wide strut (plane strain in $x_{1} z$ plane) is considered that is composed of two isotropic face sheets $\left(E_{\mathrm{f}}=70 \mathrm{GPa}, v_{\mathrm{f}}=0.3\right.$ ) and an orthotropic core with the following properties: $E_{3}^{\mathrm{c}}=110 \mathrm{MPa}, G_{13}^{\mathrm{c}}=G_{23}^{\mathrm{c}}=27 \mathrm{MPa}, G_{12}^{\mathrm{c}}=10 \mathrm{MPa}$ and $v_{i j}^{\mathrm{c}}=0$. The axial moduli of the core $E_{1}^{\mathrm{c}}=E_{2}^{\mathrm{c}}$ are defined according to the orthotropy ratio $\chi=E_{1}^{\mathrm{c}} / E_{3}^{\mathrm{c}}$ varying between $\chi=1$ (isotropic) and $\chi=10^{-6}$. In order to set the main focus on the wrinkling behavior, the strut is taken to be short $(a / h=5)$ and with a low face thickness ratio $\left(R_{\mathrm{f}}=h_{\mathrm{f}} / h=0.01\right)$. A total thickness of $h=30 \mathrm{~mm}$ has been taken for the numerical results. 

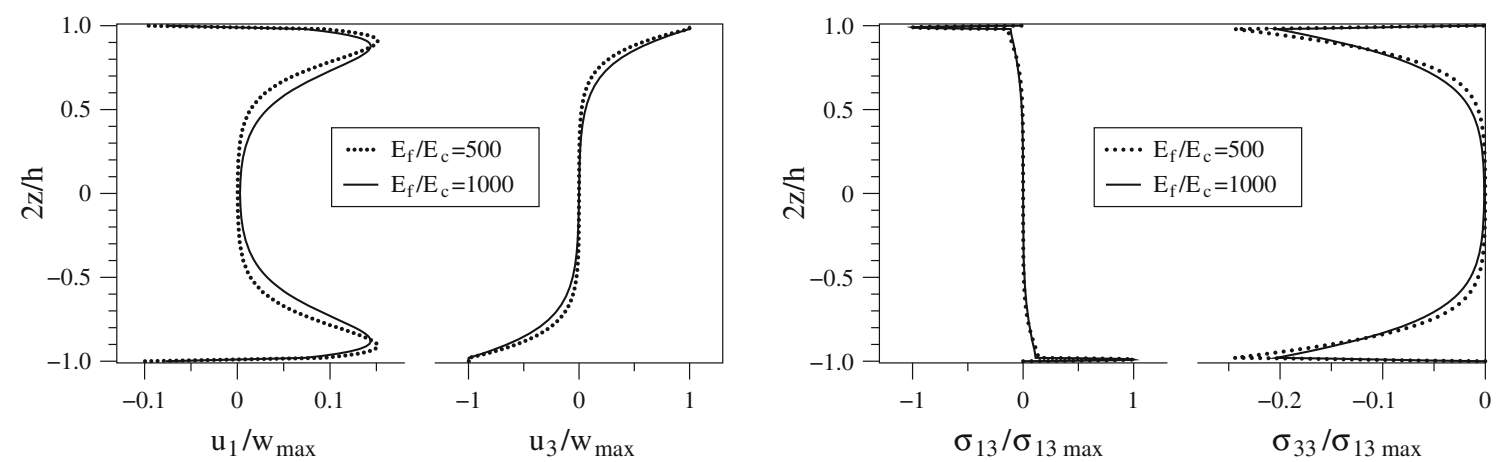

Fig. 12 Symmetric wrinkling modes of a sandwich strut with isotropic face sheets and core $\left(R_{\mathrm{f}}=0.01\right)$ for two stiffness ratios $E_{\mathrm{f}} / E_{\mathrm{c}}$ : through-thickness modal shapes of the axial and transverse displacements $u_{1}$ and $u_{3}$ (left) and of the transverse shear and normal stresses $\sigma_{13}$ and $\sigma_{33}$ (right)

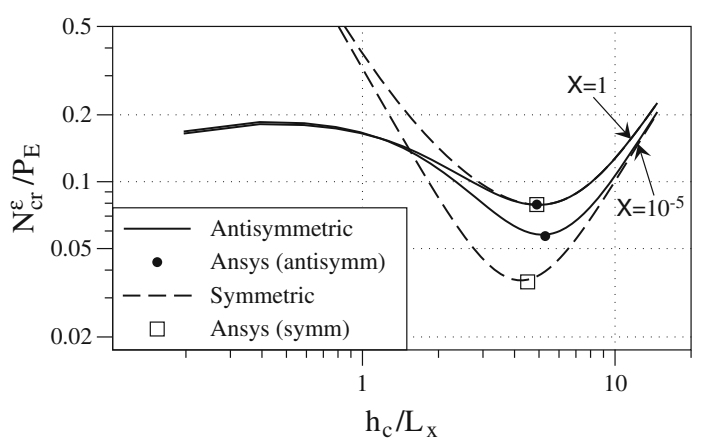

Fig. 13 Non-dimensional critical loads $N_{\mathrm{cr}}^{\epsilon} / P_{\mathrm{E}}$ versus normalized half-wavelength $h_{\mathrm{c}} / L_{x}=m\left(h_{\mathrm{c}} / a\right)$ for two values of core's orthotropy ratio $\chi=E_{1}^{\mathrm{c}} / E_{3}^{\mathrm{c}}\left(R_{\mathrm{f}}=0.01, a / h=5\right)$

Figure 13 illustrates the evolution of the non-dimensional critical loads $N_{\mathrm{cr}}^{\epsilon} / P_{\mathrm{E}}$ as a function of the halfwavelength ratio $h_{\mathrm{c}} / L_{x}$ for $\chi=1$ and $\chi=10^{-5}$. In contrast to isotropic cores, symmetric wrinkling dominates if the core's axial stiffness is low. This is in accordance with the results discussed in [30]. Furthermore, comparison with Ansys FEM results provides an excellent quantitative agreement for both symmetric and antisymmetric buckling modes. According to the model discussed in [30], the decay rate inside the core depends on the orthotropy ratio $\chi$ and diminishes as the core axial stiffness decreases. This means that the interaction between the face sheets, which entails the separation between the wrinkling loads for symmetric and antisymmetric modes, is promoted by the low axial stiffness of the core. The curves in Fig. 13 confirm this tendency showing that the separation between symmetric and antisymmetric modes is more pronounced for a low axial stiffness of the core.

Non-dimensional wrinkling loads $P^{\epsilon} / P_{\mathrm{E}}$ for varying orthotropy ratio $\chi=E_{1}^{\mathrm{c}} / E_{3}^{\mathrm{c}}$ are reported in Fig. 14 (left). An excellent agreement with FEM results obtained by Ansys is again found for the whole range of configurations. If $\chi=1$ (isotropic core), the symmetric and antisymmetric wrinkling modes occur at the same load, see also Sect. 3.3. As the axial modulus of the core gets smaller, the wrinkling load decreases and the symmetric wrinkling mode dominates. Comparison with Winkler's elastic foundation model reveals that the simple formula in Eq. (4) does accurately predict the wrinkling load for cores with very low axial stiffness. Physical insight for this finding can be caught by the analysis of the strain energy contributions for the symmetric and antisymmetric modes illustrated in Fig. 14 (right). For isotropic cores $(\chi=1)$, the total strain energy is equally distributed between the axial (bending) contribution of the face sheets and the transverse shear and normal contributions of the core. It may be interesting to note that this finding substantiates classical wrinkling formulas such as Eq. (5). As the orthotropy ratio decreases, the contribution of the transverse shear energy decreases up to a constant value of approximately $10 \%$ for the antisymmetric mode, while it gets negligible for the symmetric mode. The antisymmetric wrinkling mode is indeed always associated with a transverse shear deformation of the core, whereas in a symmetric wrinkling mode the core may undergo only extensional strains along its thickness. Winkler's model, neglecting the transverse shear deformation of the core, is thus shown to well predict the most critical buckling mode, i.e., symmetric wrinkling, of sandwich 

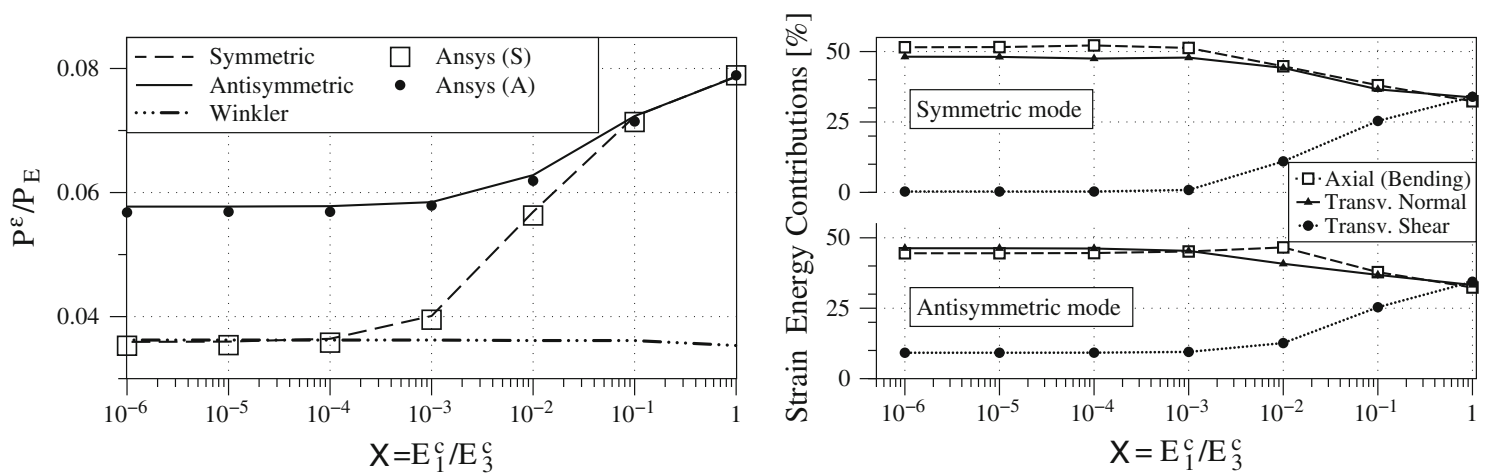

Fig. 14 Influence of core's orthotropy ratio $\chi=E_{1}^{\mathrm{c}} / E_{3}^{\mathrm{c}}$ on the non-dimensional wrinkling loads for symmetric and antisymmetric modes (left) and corresponding strain energy contributions (right) $\left(R_{\mathrm{f}}=0.01, a / h=5\right)$

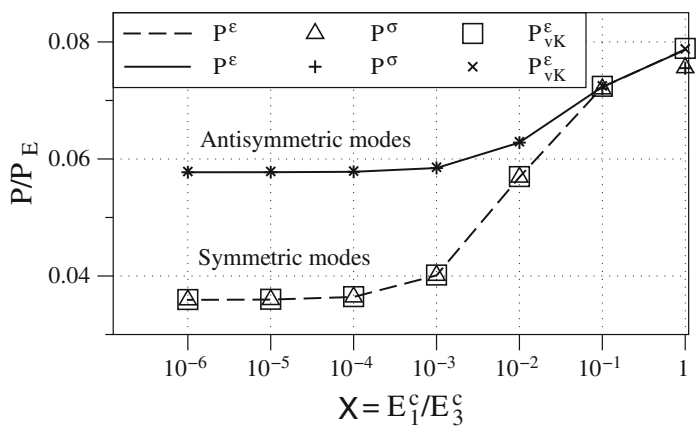

Fig. 15 Effect of von Kàrmàn nonlinearities and preload formulation on the non-dimensional buckling loads $P / P_{\mathrm{E}}\left(R_{\mathrm{f}}=\right.$ $0.01, a / h=5)$

struts with low axial stiffness. The same conclusion is reached for nonzero Poisson's ratios of the core (results not reported for brevity).

A final comment for this section concerns the comparison between buckling loads obtained by the $P^{\epsilon}$ formulation, those relying on von Kàrmàn nonlinearities $\left(P_{v K}^{\epsilon}\right)$ and those referring to a preload that is entirely carried by the face sheets $\left(P^{\sigma}\right)$. The result of this comparison is summarized in Fig. 15, which reports the buckling loads obtained by these different formulations for the symmetric and antisymmetric modes. The $P_{v K}^{\sigma}$ formulation has been excluded from the comparison because it coincides with the $P^{\sigma}$ one. It can be seen that the only (small) difference is found for the isotropic core $\chi=1$, for which the $P^{\sigma}$ load is lower than that obtained by a preload carried by the entire sandwich cross-section. No difference can be appreciated between von Kàrmàn's and the full Green-Lagrange nonlinear formulation.

\subsection{Buckling of sandwich plates with orthotropic face sheets}

This section is concerned with the stability analysis of a flat sandwich panel made of composite face sheets and an orthotropic (honeycomb) core. The plate is simply supported along its four edges and is uniaxially compressed along the $x_{1}$ axis. As a result, instability occurs with one halfwave along the $y$ direction, i.e., $n=1$, and a variable number $m$ of halfwaves along the $x_{1}$ direction. Two symmetric sandwich panels are considered with different stacking sequences for the composite face sheets made of two CFRP plies: starting from the outer surfaces, the sequences are $0^{\circ} / 90^{\circ}$ (cross-ply face sheets, panel 1 ) and $0^{\circ} / 0^{\circ}$ (unidirectional face sheets with fibers aligned along $x_{1}$ axis, panel 2). The panel is square with $a=b=228 \mathrm{~mm}$, the core thickness is $h_{\mathrm{c}}=25 \mathrm{~mm}$, and that of the face sheets is $h_{\mathrm{f}}=0.25 \mathrm{~mm}$ (each ply is $0.125 \mathrm{~mm}$ thick). The material properties for the face sheets and for the core are given in Table 4, where it is noted that an antiplane model is used for the honeycomb core. Since the panel is rather thick $(a / h \approx 9)$, the faces rather thin $\left(h_{\mathrm{f}} / h_{\mathrm{c}}=0.01\right)$ and the in-plane stiffness of the core is negligible, symmetric wrinkling is expected to be the dominant instability. 
Table 4 Material data for the wrinkling analysis of the sandwich panel

\begin{tabular}{lll}
\hline & CFRP ply & Honeycomb core \\
\hline$E_{1}(\mathrm{MPa})$ & $142 \times 10^{3}$ & 0 \\
$E_{2}(\mathrm{MPa})$ & $9.8 \times 10^{3}$ & 0 \\
$E_{3}(\mathrm{MPa})$ & $9.8 \times 10^{3}$ & 109.0 \\
$G_{23}(\mathrm{MPa})$ & $4.3 \times 10^{3}$ & 15.5 \\
$G_{13}(\mathrm{MPa})$ & $4.3 \times 10^{3}$ & 26.6 \\
$G_{12}(\mathrm{MPa})$ & $4.3 \times 10^{3}$ & 0 \\
$\nu_{i j}(-)$ & 0.34 & 0 \\
\hline
\end{tabular}

Table 5 Wrinkling loads per unit width (N/mm) for the CFRP/honeycomb panel

\begin{tabular}{|c|c|c|c|c|c|c|}
\hline & $\mathrm{P}-\mathrm{W}_{\text {Expt }}[63]$ & $\mathrm{P}-\mathrm{W}_{\text {Anlt }}[63]$ & $\mathrm{H}-\mathrm{M}$ [42] & D-D-M [46] & Y-D [64] & LM4 \\
\hline $\begin{array}{l}\text { Panel } 1 \\
\text { w/adhesive }\end{array}$ & 137 & $\begin{array}{r}77 \\
117\end{array}$ & $\begin{array}{l}77.43 \\
130.4\end{array}$ & $\begin{array}{l}76.23(49) \\
127.1(38)\end{array}$ & $76.16(49)$ & $\begin{array}{l}76.24(49) \\
127.1(38)\end{array}$ \\
\hline $\begin{array}{l}\text { Panel } 2 \\
\text { w/adhesive }\end{array}$ & 191 & 160 & $\begin{array}{l}161.3 \\
183.8\end{array}$ & $\begin{array}{l}157.6(35) \\
179.0(32)\end{array}$ & $157.6(34)$ & $\begin{array}{l}157.6(34) \\
179.0(32)\end{array}$ \\
\hline
\end{tabular}

Numbers in parentheses indicate the number of halfwaves $m$

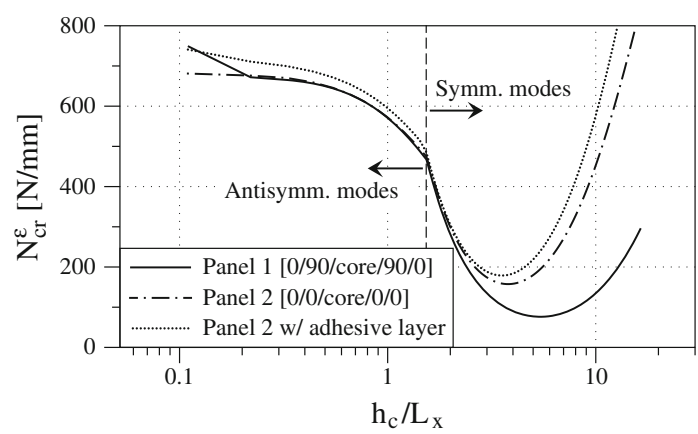

Fig. 16 Critical loads $N_{\mathrm{cr}}^{\epsilon}$ per unit width for two square panels (CFRP/honeycomb sandwich)

Experimental, analytical and numerical results for these configurations have been reported by a number of authors. Predicted wrinkling loads per unit width obtained by the present LM4 model are compared in Table 5 with the experimental results of Pearce and Webber $\left(\mathrm{P}-\mathrm{W}_{\text {Expt }}\right)$ [63], the analytical results of the same authors $\left(\mathrm{P}-\mathrm{W}_{\text {Anlt }}\right)$, the numerical results by Hadi and Matthews $(\mathrm{H}-\mathrm{M})$ [42], those by Dafedar et al. (D-D-M) [46], and those by Yuan and Dawe (Y-D) [64]. All predicted wrinkling loads are in close agreement but underestimate the experimentally measured critical load. In order to increase the wrinkling load and thus improve the correlation with the experiment, an adhesive layer between the face sheet and the core may be included in the analysis [65]: This additional layer is $0.25 \mathrm{~mm}$ thick and is isotropic with $E_{\text {adh }}=3.05 \mathrm{GPa}$ and $v_{\text {adh }}=0.3[42,46]$.

The evolution of the critical load with respect to the normalized half-wavelength $h_{\mathrm{c}} / L_{x}$ is displayed in Fig. 16. For both panels, symmetric wrinkling with a half-wavelength smaller than the core thickness is the dominant instability mode. The switch between antisymmetric and symmetric wrinkling mode occurs for $m=15$ at $h_{\mathrm{c}} / L_{x} \approx 1.5$. The panel with cross-ply face sheets (panel 1) has a higher global buckling load but a lower wrinkling load compared to the panel with unidirectional face sheets (panel 2). The presence of the adhesive layer in panel 2 enhances its global buckling load up to the level of panel 1. In conclusion, this example gives evidence of the usefulness of an accurate model capable to represent both global and local instabilities independently of the number and nature of the plies constituting the sandwich panel.

\section{Conclusions}

This paper presented a unified plate model for the linearized analysis of periodic global and local buckling of symmetric sandwich panels in the framework of Navier's solution for simply supported struts. The plate model 
is based on a partially mixed approach and a layer-wise fourth polynomial expansion, irrespective of the nature of the ply (thin or thick, isotropic or orthotropic). Comparison with elasticity results available in the literature and obtained by accurate FEM analysis has shown the quasi-3D accuracy of the model for thick and thin face sheets as well as isotropic and orthotropic materials (composite face sheets and honeycomb core). This model allows a comprehensive discussion of geometrical and constitutive parameters influencing the stability limit and the buckling wavelength as well as of the role of von Kàrmàn's nonlinearities and of the way the initial load is applied. The following conclusions can be summarized:

1. The model follows well Engesser's theory for the global buckling of shear flexible struts even if the shear crimping limit cannot be exactly recovered because of the non-periodic nature of this instability.

2. Short-wavelength buckling (wrinkling) is the dominating instability of rather short struts and is promoted by low face sheet thickness and low core stiffness.

3. The dominating local instability of sandwich struts with isotropic cores is the antisymmetric wrinkling mode, whereas symmetric wrinkling dominates when the cores are orthotropic (lower in-plane stiffness compared to transverse normal stiffness).

4. The present model shows that symmetric and antisymmetric wrinkling coincide in the short-wavelength regime, which is promoted by thick and stiff cores.

5. Energy considerations provided a sound justification of the classical wrinkling formula and of the applicability of the simple Winkler model for anti-plane cores.

6. No differences have been noticed in the global buckling prediction when von Kàrmàn's nonlinearities are used or if the initial load is assumed to be carried only by the thin face sheets. On the contrary, the wrinkling loads of sandwich struts with low $E_{\mathrm{f}} / E_{\mathrm{c}}$ ratios are higher if the initial load is carried not only by the face sheets but by the core as well.

\section{Appendix}

According to the compact formulation employed in this paper and extensively reported, e.g., in [55], the eigenvalue problem related to the linearized bifurcation buckling analysis can be cast in terms of kernels as reported in Eq. (17). These arrays of dimension $3 \times 3$ are the building blocks of the plate model in that they represent the elementary contribution related to each layer (index $k$ ) and to each term of the through-thickness expansion of the virtual variation (index $\tau$ ) and the actual unknown (index $s$ ), see Eq. (15). This Appendix reports the nonzero entries of these kernels, where the subscript $(m n)$ can be omitted without loss of clarity. After introducing the integrals over the layer's thickness $h_{k}$ as follows:

$$
J^{k \tau s}=\int_{h_{k}} F_{\tau}(z) F_{s}(z) \mathrm{d} z ; \quad J^{k \tau_{z} s}=\int_{h_{k}} \frac{\mathrm{d} F_{\tau}(z)}{\mathrm{d} z} F_{s}(z) \mathrm{d} z,
$$

the nonzero entries of the $3 \times 3$ kernels $\mathbf{K}_{U U}^{k \tau s}, \mathbf{K}_{U T}^{k \tau s}$ and $\mathbf{K}_{T T}^{k \tau s}$ that constitute the linear stiffness matrix are:

$$
\begin{gathered}
K_{U U 11}^{k \tau s}=J^{k \tau s}\left(m^{2} \tilde{C}_{11}^{k}+n^{2} \tilde{C}_{66}^{k}\right) ; \quad K_{U U 22}^{k \tau s}=J^{k \tau s}\left(m^{2} \tilde{C}_{66}^{k}+n^{2} \tilde{C}_{22}^{k}\right) ; \\
K_{U U 12}^{k \tau s}=K_{U U 21}^{k \tau s}=m n J^{k \tau s}\left(\tilde{C}_{12}^{k}+\tilde{C}_{66}^{k}\right) ; \\
K_{U T 11}^{k \tau s}=K_{U T 22}^{k \tau s}=K_{U T 33}^{k \tau s}=J^{k \tau \tau_{z} s} ; \quad K_{U T 13}^{k \tau s}=-m \tilde{C}_{13} J^{k \tau s} ; \\
K_{U T 23}^{k \tau s}=-n \tilde{C}_{23} J^{k \tau s} ; \quad K_{U T 31}^{k \tau s}=m J^{k \tau s} ; \quad K_{U T 32}^{k \tau s}=n J^{k \tau s} . \\
K_{T T 11}^{k \tau s}=-\tilde{C}_{55}^{k \tau s} J^{k \tau s} ; \quad K_{T T 22}^{k \tau s}=-\tilde{C}_{44} J^{k \tau s} ; \quad K_{T T 33}^{k \tau s}=-\tilde{C}_{33} J^{k \tau s} .
\end{gathered}
$$

As far as the geometric stiffness matrix $\mathbf{K}_{\mathrm{g}}^{k \tau s}$ is concerned, a tracer $\delta_{v K}$ is used in order to introduce von Kàrmàn's approximation, through which some terms of the nonlinear strain field are set to zero. For a unit compressive stress, uniform across the thickness of the $k$ th layer, the nonzero terms of $\mathbf{K}_{\mathrm{g}}^{k \tau s}$ read

$$
\mathbf{K}_{\mathrm{g}}^{k \tau s}(1,1)=\mathbf{K}_{\mathrm{g}}^{k \tau s}(2,2)=-\delta_{v K} m^{2} J^{k \tau s} ; \quad \mathbf{K}_{\mathrm{g}}^{k \tau s}(3,3)=-m^{2} J^{k \tau s} .
$$

If the pre-buckling state is given by a unit compressive strain, uniform across the thickness of the $k$ th layer, the nonzero terms of $\mathbf{K}_{g}^{k \tau s}$ have the following expressions:

$$
\mathbf{K}_{\mathrm{g}}^{k \tau s}(1,1)=\mathbf{K}_{\mathrm{g}}^{k \tau s}(2,2)=-\delta_{v K} m^{2} C_{11}^{k} J^{k \tau s} ; \quad \mathbf{K}_{\mathrm{g}}^{k \tau s}(3,3)=-m^{2} C_{11}^{k} J^{k \tau s} .
$$




\section{References}

1. Vizzini, A.J.: Lightweight sandwich structures. In: Blockley, R., Shyy, W. (eds.) Encyclopedia of Aerospace Engineering, Wiley, New York (2010)

2. Davies, J.M. (eds.): Lightweight Sandwich Construction. Blackwell Science Ltd., Oxford (2001)

3. Carlsson, L.A., Kardomateas, G.A.: Structural and Failure Mechanics of Sandwich Composites. Springer, Berlin (2011)

4. Daniel, I.M., Gdoutos, E.E., Wang, K.-A., Abot, J.L.: Failure modes of composite sandwich beams. Int. J. Damage Mech. 11, 309-334 (2002)

5. Vadakke, V., Carlsson, L.A.: Experimental investigation of compression failure mechanisms of composite faced foam core sandwich specimens. J. Sandw. Struct. Mater. 6, 327-342 (2004)

6. Fleck, N.A., Sridhar, I.: End compression of sandwich columns. Compos. A 33, 353-359 (2002)

7. Fagerberg, L.: Wrinkling and compression failure transition in sandwich panels. J. Sandw. Struct. Mater. 6, 129-144 (2004)

8. Sullins, R.T., Smith, G.W., Spier, E.E.: Manual for Structural Stability Analysis of Sandwich Plates and Shells. Technical Report NASA CR-1457, NASA (1969)

9. ESA-ESTEC (eds). ECSS-E-HB-32-24A-Buckling of Structures. ESA Requirements and Standards Division, Noordwijk, The Netherlands (2010)

10. Ley, R.P., Lin, W., Mbanefo, U.: Facesheet Wrinkling in Sandwich Structures. Technical Report NASA/CR-1999-208994, NASA (1999)

11. Staal, R.A., Mallinson, G.D., Jayaraman, K., Horrigan, D.P.W.: Predicting failure loads of undamaged sandwich honeycomb panels subject to bending. J. Sandw. Struct. Mater. 11, 73-104 (2009)

12. Wadsworth, D.J., Horrigan, D.P.W., Moltschaniwskyi, G., Collins, I.: Facesheet wrinkling of damaged honeycomb sandwich structures. J. Sandw. Struct. Mater. 11, 105-131 (2009)

13. Hunt, G.W., Wadee, M.A.: Localization and mode interaction in sandwich structures. Proc. R. Soc. Lond. A 454, 1197$1216(1998)$

14. Léotoing, L., Drapier, S., Vautrin, A.: Nonlinear interaction of geometrical and material properties in sandwich beam instabilities. Int. J. Solids Struct. 39, 3717-3739 (2002)

15. Coman, C.D.: On interactive buckling in a sandwich structure. ZAMP 61, 565-577 (2010)

16. Hu, H., Belouettar, S., Potier-Ferry, M., Makradi, A., Koutsawa, Y.: Assessment of various kinematic models for instability analysis of sandwich beams. Eng. Struct. 2011, 572-579 (2011)

17. Rammerstorfer, F.G., Pahr, D.H., Daxner, T., Vonach, W.K.: Buckling in thin walled micro and meso structures of lightweight materials and material compounds. Comput. Mech. 37, 470-478 (2006)

18. Yu, K., Hu, H., Chen, S., Belouettar, S., Potier-Ferry, M.: Multi-scale techniques to analyze instabilities in sandwich structures. Compos. Struct. 96, 751-762 (2013)

19. Humer, A.: Exact solutions for the buckling and postbuckling of shear-deformable beams. Acta Mech. 224, 1493-1525 (2013)

20. Norris, C.B., Ericksen, W.E., March, H.W., Smith, C.B., Boller, K.H.: Wrinkling of the Facings of Sandwich Construction Subjected to Edgewise Compression. Technical Report 1810, Forest Products Laboratory (1961)

21. Frostig, Y., Baruch, M.: High-order buckling analysis of sandwich beams with transversely flexible core. J. Eng. Mech. 119, 476-495 (1993)

22. Frostig, Y.: Buckling of sandwich panels with a flexible core-high-order theory. Int. J. Solids Struct. 35, 183-204 (1998)

23. Fagerberg, L., Zenkert, D.: Imperfection-induced wrinkling material failure in sandwich panels. J. Sandw. Struct. Mater. 7, 195-219 (2005)

24. Beghini, A., Bažant, Z.P., Waas, A.M., Basu, S.: Postcritical imperfection sensitivity of sandwich or homogenized orthotropic columns soft in shear and in transverse deformation. Int. J. Solids Struct. 43, 5501-5524 (2006)

25. Hohe, J., Librescu, L.: Recent results on the effect of the transverse core compressibility on the static and dynamic response of sandwich structures. Compos. B 39, 108-119 (2008)

26. Allen, H.G.: Analysis and Design of Structural Sandwich Panels. Pergamon Press, Oxford (1969)

27. Bažant, Z.P., Beghini, A.: Sandwich buckling formulas and applicability of standard computational algorithm for finite strain. Compos. B 35, 573-581 (2004)

28. Kardomateas, G.A.: An elasticity solution for the global buckling of sandwich beams/wide panels with orthotropic phases. J. Appl. Mech. 77, 021015.1-021015.7 (2010). doi:10.1115/1.3173758

29. Niu, K., Talreja, R.: Modeling of wrinkling in sandwich panels under compression. J. Eng. Mech. 125, 875-883 (1999)

30. Vonach, W.K., Rammerstorfer, F.G.: The effects of in-plane core stiffness on the wrinkling behavior of thick sandwiches. Acta Mech. 141, 1-10 (2000)

31. Thomsen, O.T.: Analysis of local bending effects in sandwich plates with orthotropic face layers subjected to localised loads. Compos. Struct. 25, 511-520 (1993)

32. Aiello, M.A., Ombres, L.: Local buckling loads of sandwich panels made with laminated faces. Compos. Struct. 38, 191201 (1997)

33. Navarro, P., Abrate, S., Aubry, J., Marguet, S., Ferrero, J.-F.: Analytical modeling of indentation of composite sandwich beam. Compos. Struct. 100, 79-88 (2013)

34. Zenkour, A.M., Allam, M.N.M., Shaker, M.O., Radwan, A.F.: On the simple and mixed first-order theories for plates resting on elastic foundations. Acta Mech. 220, 33-46 (2011)

35. Hoff, N.J., Mautner, S.E.: Buckling of sandwich type panels. J. Aeronaut. Sci. 12, 285-297 (1945)

36. Plantema, F.J.: Sandwich Construction. Wiley, New York (1966)

37. Boeing Design Manual BDM-6718.: Face Wrinkling of Flat Honeycomb Sandwich Panels with Isotropic Faces (1989)

38. Birman, V., Bert, C.W.: Wrinkling of composite-facing sandwich panels under biaxial loading. J. Sandw. Struct. Mater. 6, 217237 (2004)

39. Koissin, V., Shipsha, A., Skvortsov, V.: Wrinkling in sandwich panels-an analytical approach. J. Sandw. Struct. Mater. 13, 705-730 (2011) 
40. Hohe, J., Librescu, L.: Advances in the structural modeling of elastic sandwich panels. Mech. Adv. Mater. Struct. 11, 395424 (2004)

41. Benson, A.S., Mayers, J.: General instability and face wrinkling of sandwich plates—unified theory and applications. AIAA J. 5, 729-739 (1967)

42. Hadi, B.K., Matthews, F.L.: Development of Benson-Mayers theory on the wrinkling of anisotropic sandwich panels. Compos. Struct. 49, 425-434 (2000)

43. Léotoing, L., Drapier, S., Vautrin, A.: First applications of a novel unified model for global and local buckling of sandwich columns. Eur. J. Mech. Solids 21, 683-701 (2002)

44. Ji, W., Waas, A.M.: Global and local buckling of a sandwich beam. J. Eng. Mech. 133, $230-237$ (2007). doi:10.1061/ (ASCE)0733-9399(2007)133:2(230)

45. Douville, M.-A., Le Grognec, P.: Exact analytical solutions for the local and global buckling of sandwich beam-columns under various loadings. Int. J. Solids Struct. 50, 2597-2609 (2013)

46. Dafedar, J.B., Desai, Y.M., Mufti, A.A.: Stability of sandwich plates by mixed, higher-order analytical formulation. Int. J. Solids Struct. 40, 4501-4517 (2003)

47. Meyer-Piening, H.-R.: A refined theory for the analysis of sandwich beams and its application to local and global stability investigations. In: Sandwich Structures: Analysis and Design Issues for Modern Aerospace Vehicles, pp. 379-386. ASME (1997)

48. Meyer-Piening, H.-R.: Experiences with "exact" linear sandwich beam and plate analyses regarding bending, instability and frequency investigations. In: Proceedings of the Fifth International Conference on Sandwich Constructions, vol. 2, pp. 705-716. Zurich, Switzerland (2000)

49. Kardomateas, G.A.: Wrinkling of wide sandwich panels/beams with orthotropic phases by an elasticity approach. J. Appl. Mech. 72, 818-825 (2005)

50. Ji, W., Waas, A.M.: Wrinkling and edge buckling in orthotropic sandwich beams. J. Eng. Mech. 134, 455-461 (2008). doi:10.1061/(ASCE)0733-9399(2008)134:6(455)

51. Ji, W., Waas, A.M.: 2D elastic analysis of the sandwich panel buckling problem: benchmark solutions and accurate finite element formulations. ZAMP 61, 897-917 (2009)

52. Reissner, E.: On a certain mixed variational theorem and a proposed application. Int. J. Numer. Methods Eng. 20, 1366$1368(1984)$

53. Carrera, E.: Developments, ideas and evaluations based upon Reissner's mixed variational theorem in the modeling of multilayered plates and shells. Appl. Mech. Rev. 54, 301-329 (2001)

54. Carrera, E.: An assessment of mixed and classical theories on global and local response of multilayered orthotropic plates. Compos. Struct. 50, 183-198 (2000)

55. Carrera, E.: Theories and finite elements for multilayered plates and shells: a unified compact formulation with numerical assessment and benchmarking. Arch. Comput. Methods Eng. 10, 215-296 (2003)

56. Ciuffreda, A., Carrera, E.: Bending of composites and sandwich plates subjected to localized lateral loadings: a comparison of various theories. Compos. Struct. 68, 185-202 (2005)

57. Demasi, L.: 2D, quasi 3D and 3D exact solutions for bending of thick and thin sandwich plates. J. Sandw. Struct. Mater. 10, 231-271 (2008)

58. Carrera, E., Brischetto, S.: A survey with numerical assessment of classical and refined theories for the analysis of sandwich plates. Appl. Mech. Rev. 62, 1-17 (2009)

59. D’Ottavio, M., Carrera, E.: Variable-kinematics approach for linearized buckling analysis of laminated plates and shells. AIAA J. 48, 1987-1996 (2010)

60. Washizu, K.: Variational Methods in Elasticity and Plasticity, 2 edn. Pergamon Press, Oxford (1975)

61. Reddy, J.N.: Mechanics of Laminated Composite Plates and Shells: Theory and Analysis, 2 edn. CRC Press, Boca Raton, FL (2004)

62. Carrera, E., Demasi, L.: Classical and advanced multilayered plate elements based upon PVD and RMVT. Part 1: derivation of finite element matrices. Int. J. Numer. Methods Eng. 55, 191-231 (2002)

63. Pearce, T.R.A., Webber, J.P.H.: Experimental buckling loads of sandwich panels with carbon fibre faceplates. Aeronaut. Q. 24, 295-312 (1973)

64. Yuan, W.X., Dawe, D.J.: Overall and local buckling of sandwich plates with laminated faceplates, part II: applications. Comput. Methods Appl. Mech. Eng. 190, 5215-5231 (2001)

65. Webber, J.P.H., Kyriakides, S., Lee, C.T.: On the wrinkling of honeycomb sandwich columns with laminated cross-ply faces. Aeronaut. J. 80, 264-272 (1976) 